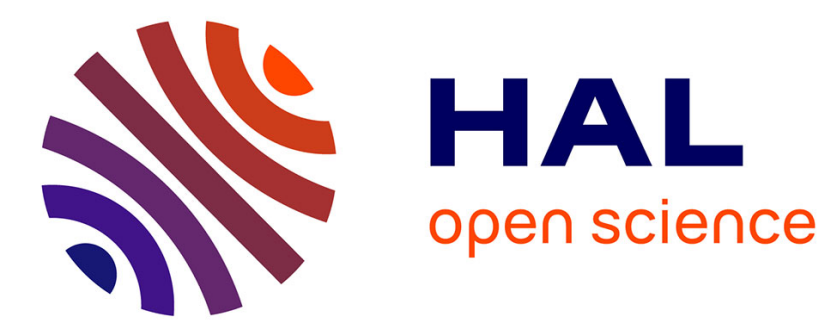

\title{
Chalcogenide glasses for innovation in applied science: fundamental issues and new insights
}

Carlo Massobrio, Assil Bouzid, Mauro Boero, Guido Ori, Évelyne Martin, Sébastien Le Roux

\section{To cite this version:}

Carlo Massobrio, Assil Bouzid, Mauro Boero, Guido Ori, Évelyne Martin, et al.. Chalcogenide glasses for innovation in applied science: fundamental issues and new insights. Journal of Physics D: Applied Physics, 2020, 53 (3), pp.033002. 10.1088/1361-6463/ab48a4 . hal-02349413

\section{HAL Id: hal-02349413 https://hal.science/hal-02349413}

Submitted on 23 Nov 2020

HAL is a multi-disciplinary open access archive for the deposit and dissemination of scientific research documents, whether they are published or not. The documents may come from teaching and research institutions in France or abroad, or from public or private research centers.
L'archive ouverte pluridisciplinaire HAL, est destinée au dépôt et à la diffusion de documents scientifiques de niveau recherche, publiés ou non, émanant des établissements d'enseignement et de recherche français ou étrangers, des laboratoires publics ou privés. 


\title{
Chalcogenides glasses for innovation in applied science: fundamental issues and new insights
}

\author{
Carlo Massobrio, ${ }^{1}$ Assil Bouzid, ${ }^{2}$ Mauro Boero, ${ }^{1}$ \\ Guido Ori, ${ }^{1}$ Evelyne Martin, ${ }^{3}$ and Sébastien Le Roux ${ }^{1}$ \\ ${ }^{1}$ Université of Strasbourg, Institut de Physique et de Chimie des Matériaux de Strasbourg, \\ 23 rue du Loess, BP43, F-67034 Strasbourg Cedex 2, France \\ ${ }^{2}$ Chaire de Simulation à l'Echelle Atomique (CSEA), \\ Ecole Polytechnique Fédérale de Lausanne (EPFL), CH-1015, Lausanne, Switzerland \\ ${ }^{3}$ Université de Lille, CNRS, Centrale Lille, ISEN, \\ Université de Valenciennes, UMR 8520 - IEMN, F-59000 Lille, France
}

(Dated: September 16, 2019)

\begin{abstract}
We provide an overview of what has been accomplished by our team, since 1998, in the area of firstprinciples molecular dynamics (FPMD) modeling of glassy chalcogenides, a prototypical family of network disordered materials. After a broad introduction that defines the main motivation and strategy of our approach, we treat several cases by focusing first on a specific issue of generic character (the origin of the first sharp diffraction peak in the neutron structure factor as a signature of intermediate range order) and then on particular systems mostly within (or closely related to) the $\mathrm{Ge}_{\mathrm{x}} \mathrm{Se}_{1-\mathrm{x}}$ binary family. Chalcogenides ternary glasses will also be considered. It appears that first-principle molecular dynamics has acquired an unprecedented level of reliability in the field of glassy chalcogenides, by enriching and complementing successfully the knowledge of these materials.
\end{abstract}




\section{INTRODUCTION}

This contribution describes atomic-scale studies devoted to a specific class of network glasses, glassy chalcogenides, for which extensive first-principles molecular dynamics (FPMD) work has been performed by our team on some $\mathrm{Ge}_{\mathrm{x}} \mathrm{Se}_{1-\mathrm{x}}$ binary systems and ternary compounds. Focusing on disordered chalcogenides, the experience collected over the past 25 years led us to the conviction that the use of molecular dynamics in conjunction with density functional theory (DFT) is the method of choice to circumvent the limitations of effective interatomic potentials (termed also force-fields schemes or classical potentials in what follows). Classical potentials are intrinsically unable to describe deviations from chemically ordered topologies occurring, for a given stoichiometry, in specific network structures (as glassy chalcogenides) made of interconnected tetrahedra. This issue can be better understood by considering the case of a model $\mathrm{AX}_{2}$ system featuring a marked difference of electronegativity between $\mathrm{A}$ and $\mathrm{X}$ atomic species $\left(\mathrm{SiO}_{2}\right.$ being a valuable example, electronegativity $\mathrm{Si}=1.9, \mathrm{O}=3.44)$. The simple assignment of formal negative and positive point charges ensures the establishment of a tetrahedral network resulting in structural properties broadly consistent with experiments. However, problems occur when high temperatures come into play or when, by changing the chemical composition, the differences of electronegativity are smaller (for instance, in $\mathrm{GeSe}_{2}$, electronegativity $\mathrm{Ge}=2.01, \mathrm{Se}=2.55$ ). In these cases, the charge point approximation inherent in force-fields schemes becomes devoid of any physical meaning. These situations result in the emergence of structural defects (homopolar bonds and coordination other than four for A-elements and two for X-elements) that are indicative of a delicate interplay between different bonding characters (typically ionic and covalent), requiring models based on the explicit account of the electronic structure. In this review we retrace the genesis and development of our approach by describing achievements obtained when applying FPMD to disordered chalcogenides, mostly, but not exclusively, belonging to the $\mathrm{Ge}_{\mathrm{x}} \mathrm{Se}_{1-\mathrm{x}}$ family. With no loss of generality, some concepts and ideas developed hereafter stem from calculations and results obtained for liquids also. For instance, we shall review a comparative study based on FPMD and a classical potential proposed for liquid $\mathrm{GeSe}_{2}$. Overall, our main intent is to highlight different milestone results that contributed to an understanding of network chalcogenide glasses (fostering innovation in material science) well beyond the particular cases addressed.

Computational material science makes available several ways of complementing and enriching experiments. The most popular consists in calculating properties to be directly compared to ex- 
periments, by providing additional information not accessible to measurements. As an alternative instead, one can focus on a single property to extract general hints applicable to other systems close or dissimilar in nature to the original object of the study. In 1995, when we began working in this area, we selected this second route, being very much intrigued by the interplay between atomic scale structural motifs and specific marks recorded in experimental data. This guided our work toward the understanding of the origins of the first sharp diffraction peak (FSDP) in the total and in the partial structure factor of liquid $\mathrm{GeSe}_{2}$. Measurements of the partial structure factors had revealed the occurrence of a very intense FSDP in the Faber-Ziman (FZ) [1] Ge-Ge partial structure factor and in the Bhatia-Thornton (BT) [2] concentration-concentration structure factor of liquid $\mathrm{GeSe}_{2}$. We recall that the $\mathrm{FZ}$ and the BT formalisms express a description of the network based on the notion of atoms and number/concentration, respectively $[1,2]$. The existence of correlations extending well beyond nearest neighbors and reflecting fluctuations of concentration on intermediate scale was taken as a challenge for atomic-scale modeling. Two main reasons are worth invoking. First, the case of liquid $\mathrm{GeSe}_{2}$ was essentially unique among network-forming disordered materials, the FSDP in $\mathrm{S}_{\mathrm{CC}}$ being among the most prominent ever recorded. Second, such peak was absent in any available FPMD model, and this regardless of the kind of exchangecorrelation functional employed within density functional theory [3]. This last point deserves some more consideration. We were able to demonstrate in the case of liquid $\mathrm{GeSe}_{2}$ that a correct reproduction of experimental data in reciprocal space can be obtained provided the generalized gradient approximation (GGA, in one of the standard, available forms, due to Perdew and Wang (PW) [4]) is employed in the expression of the exchange correlation (XC) functional [5]. GGA is able to enhance the ionic character of bonding by ensuring the stability of the tetrahedral network. Among the GGA schemes, the one by Becke, Lee, Yang, and Parr (BLYP) [6, 7] was found to be the best performing in the case of the disordered $\mathrm{Ge}_{\mathrm{x}} \mathrm{Se}_{1-\mathrm{x}}$ family, in spite of the fact that our first results on liquid $\mathrm{GeSe}_{2}$ were obtained with the PW scheme [3]. The robust modeling framework thereby established (FPMD with BLYP-GGA) allowed us to achieve a series of results featuring quantitative predictions for disordered chalcogenides. Some of them will be described in this review, by exemplifying the link between modeling, material science and statistical mechanics as the guideline reflecting the strategy followed ever since our early involvement in this field. All the GGA schemes are implemented in the CPMD code [8] according to the scheme proposed by White and Bird [9] exploiting the fully parallelized fast Fourier transform (FFT) algorithm. In recent versions (presently the 4.3) of this same code, the FFT can be avoided by a direct real space 
implementation as proposed by Bylander and Kleinman [10], thereby allowing for a substantial speed up in the calculation performances.

\section{Atomic-scale origin of the first sharp diffraction peak}

To substantiate the present review with a triggering case marking our impact on the area of glassy chalcogenides modeling, it is worthwhile to start by recalling our ideas and results in regard to the atomic-scale understanding of the FSDP in the total structure factor and in the concentrationconcentration $\mathrm{S}_{\mathrm{CC}}$ partial structure factor. Our first insight was proposed in 2001 (see Ref. 11, 12). We started from the consideration that the relationship between the FSDP in reciprocal space and specific structural arrangements in real space had been the object of interpretations for a long time. Among the schemes frequently invoked, we can mention the one that associates the FSDP to a precise signature of crystalline-like layers, a relationship existing between the position of the FSDP and the interlayer separation [13]. A second idea is based on the occurrence of low density regions in glasses. In this framework, clusters are conjectured to be structural units having interstitial voids as neighbors. The existence of these two motifs results in correlation distances responsible for the establishment of intermediate range order. [14] We have exploited a set of criteria to ascertain whether or not the formation of layers and/or the presence of correlations between clusters and voids can be at the very origin of the FSDP in disordered network-forming systems. We were able to show that the appearance of the FSDP cannot be ascribed to crystallinelike layers, whereas cluster-voids correlations are common to all systems considered. In fact, this latter kind of correlation occurs also for models of liquids that do not feature any FSDP.

In 2007 (see Ref. 15), we put forth an interpretation for the origins of the FSDP in the Bhatia-Thornton concentration-concentration structure factor, $\mathrm{S}_{\mathrm{CC}}$ in terms of a particular kind of structural unit. Chains of two fourfold rings were found to account for the occurrence of the FSDP in $\mathrm{S}_{\mathrm{CC}}$ (see figure 1).

To achieve this conclusion, we partitioned instantaneous atomic configurations occurring during the motion of a model liquid in two sets, corresponding to high values and low values of the FSDP height. The chains of two fourfold rings are much more numerous in the configuration corresponding to high FSDP in $\mathrm{S}_{\mathrm{CC}} \cdot[15]$ 
a)

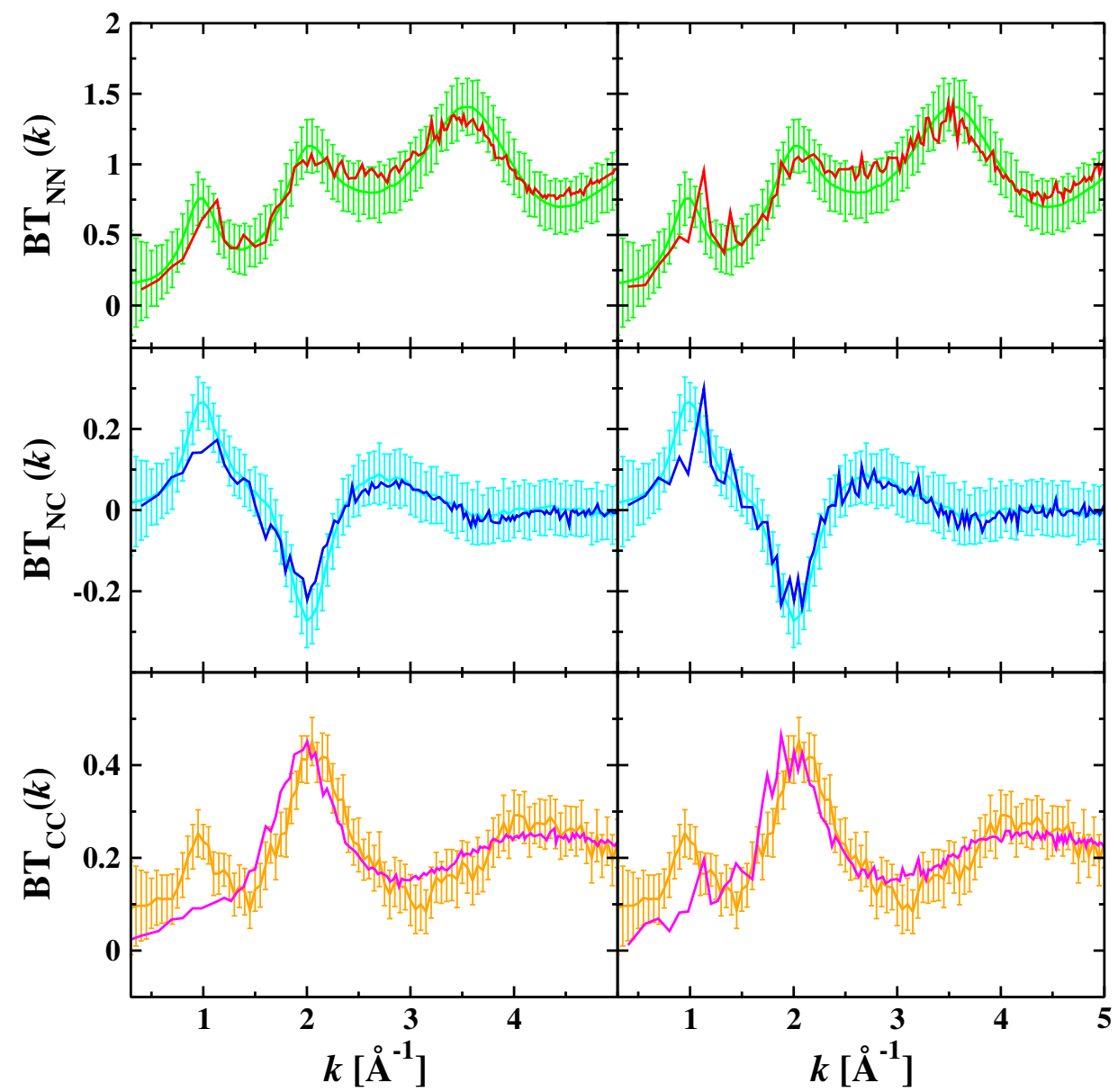

b)

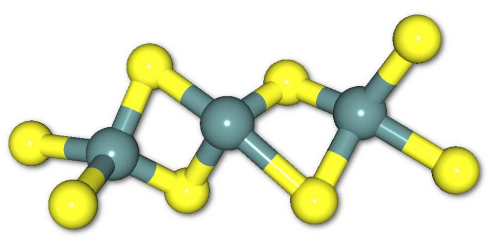

Figure 1: (Color online) a) Bhatia-Thornton partial structure factors for liquid $\mathrm{GeSe}_{2}$ : experimental measurements from Ref. 16 (lines with error bars) are compared to the results of the FPMD calculations from Ref. 15 (solid lines): from top to bottom Number-Number (NN), Number-Concentration (NC) and Concentration-Concentration (CC) partial structure factors are presented. The number of chains made of two fourfold rings (see an illustration of this motif at the bottom of the figure in b)) is much larger in the trajectories corresponding to the right column averaged data. At the same time (again, data in the right column), a distinct peak at the FSDP location is observed in the Concentration-Concentration (CC) partial structure factor. 
So far we have reported on a specific structural feature and its atomic-scale counterpart. This means that we have taken FPMD as a mean to link a signature of intermediate range order to a suitable structural motif. In what follows, we illustrate how the FSDP methodology was put to good use to unravel structural properties for a prototypical family of glassy networks. Accordingly, atomic-scale computer experiments are employed to extract, for a given system, all kinds of possible information (a priori including also the one on the electronic structure). Throughout the next part of this contribution, it has to be intended altogether that our models have been successfully validated and bear a very high predictive power. The essence of the underlying methodology has been detailed in several recent contributions devoted to the implementation of first-principles molecular dynamics, to which we refer the interested reader $[17,18]$.

\section{OVERVIEW OF ACHIEVEMENTS IN THE FPMD MODELING OF NETWORK-FORMING GLASSES}

$$
\text { Glassy } \mathrm{GeSe}_{2}
$$

The first glassy system we considered was $\mathrm{GeSe}_{2}$ (see Refs. 19, 20). We were stimulated by the existence of the partial structure factors measured by the method of isotopic substitution in neutron diffraction [21]. By producing several independent trajectories via quench from the liquid state we obtained a description of this glass consistent with experiments, even though Ge-Ge correlations features some non-negligible differences between theory and experiments. The question arises on whether such discrepancy could be ascribed to the cooling rates employed, of the order of $10^{13} \mathrm{~K} / \mathrm{s}$. It appears that the validity of this conjecture cannot be checked on appropriate, much longer time intervals, due to the intrinsic computational limits of our approach in terms of lengths of the trajectories. However, as a first observation, we point out that the reduced number of defects (i.e. the reduced departure from chemical disorder) at the end of the cooling process indicates that cooling rates as high as $10^{13} \mathrm{~K} / \mathrm{s}$ do not hamper the adjustment of the system to changes of temperature, since non negligible relaxations of the structure can occur even on very short (few ps) time scales. As a second observation, it is worthwhile to mention that a much better agreement for the Ge-Ge pair correlation functions was obtained when accounting for pressure effects,[22] so as to eliminate the residual pressures existing on the simulation periodic cell considered in Refs. 19, 20 at fixed density. This means that any concern for the possible impact of very high quench rates 
on the structure of glasses does not have to overshadow the proper account of other effects more closely related to the validity of the model. These are related to the quality of the interaction scheme and to the correct consideration of the thermodynamic conditions. Keeping these considerations in mind, our analysis of the structure of glassy $\mathrm{GeSe}_{2}$ goes as follows. Pair correlation functions are in very satisfactory agreement with the experiments in the Ge-Se and Se-Se cases. The quantity of Se-Se homopolar bonds and the number of Ge-Se neighbors are slightly larger than in the experimental counterpart. The case of Ge-Ge correlations is quite different, confirming results obtained for liquid $\mathrm{GeSe}_{2}$ [3]. As a first observation (see figure 2), the profile of $\mathrm{g}_{\mathrm{GeGe}}^{\text {th }}(r)$ lacks a well-defined minimum between $\mathrm{r}<4.5 \AA$ and $4.5 \AA<\mathrm{r}<8 \AA$.

Second, we record an underestimate for the counting of homopolar Ge-Ge bonds. This means that most of the Ge-Ge pairs found in the liquid disappear after the quenching process. Glassy $\mathrm{GeSe}_{2}$ is more chemically ordered than liquid $\mathrm{GeSe}_{2}$ One has $75.6 \%$ of Ge atoms fourfold coordinated and $93.6 \%$ of Se atoms twofold coordinated. Concerning the bonding units, we found $\mathrm{Ge}-(\mathrm{Se}), \mathrm{Ge}-\left(\mathrm{Se}_{2}\right), \mathrm{Ge}-\left(\mathrm{Se}_{3}\right), \mathrm{Ge}-\left(\mathrm{GeSe}_{3}\right), \mathrm{Ge}-\left(\mathrm{Se}_{4}\right)$ units for $\mathrm{Ge}$, and $\mathrm{Se}-(\mathrm{Ge}), \mathrm{Se}-\left(\mathrm{Se}_{2}\right), \mathrm{Se}-\left(\mathrm{Ge}_{2}\right)$, $\mathrm{Se}-\left(\mathrm{Ge}_{3}\right)$ units for Se. As anticipated above, this picture of Ge-Ge correlations was performed on a simulation cell at fixed density taken to be equal to the experimental value. Later on, as detailed in Ref. 22, we were able to demonstrate that a substantial improvement can be obtained by releasing the residual pressure acting on the system (1GPa), while keeping the basic features of the network unaltered. Finally, when comparing theory and experiments, it is worthwhile to wonder how chemical disorder establishes in the network, for instance, are there units departing from the tetrahedral coordination or preference for homopolar bonds? We found that Ge atoms prefer to form coordination groups other than $\mathrm{GeSe}_{4}$ rather than organizing themselves in $\mathrm{Ge}-\mathrm{Ge}$ dimers or chains.

\section{Glassy $\mathrm{SiSe}_{2}$}

In 2003 the structure of glassy $\mathrm{SiSe}_{2}$ was investigated in the search of a quantitative counterpart to neutron diffraction and MAS-NMR data (see Ref. 23). Our main finding was the evidence provided on glassy $\mathrm{SiSe}_{2}$ being predominantly chemically ordered, with most of the $\mathrm{Si}$ atoms in ES (edge-sharing) connections.

The network can be described as made of Si atoms lying in two, one or zero fourfold rings, respectively labeled $\operatorname{Si}(2), \operatorname{Si}(1)$ and $\operatorname{Si}(0)$, as shown in figure 3 . 


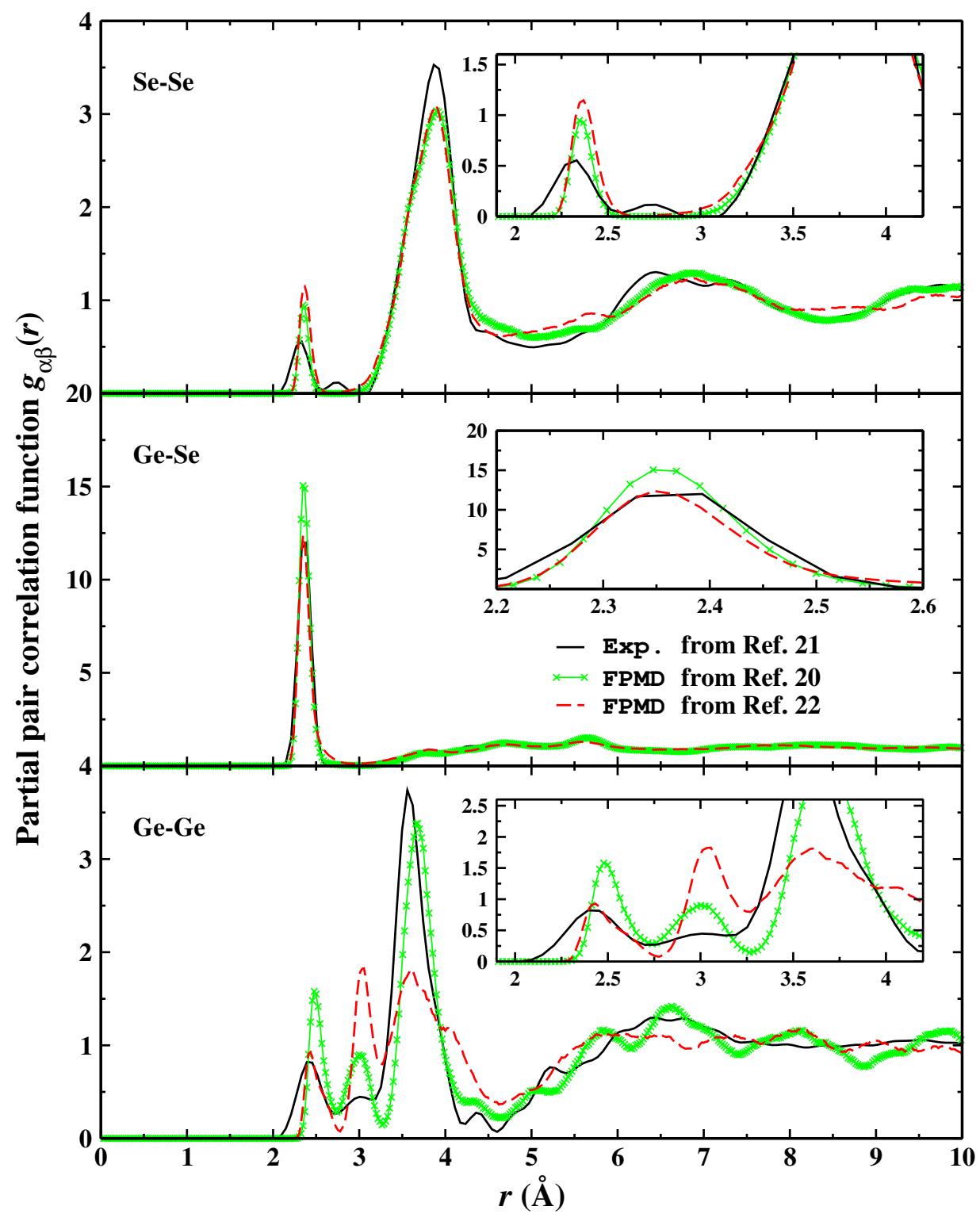

Figure 2: (Color online) Partial pair correlation function $g_{\alpha \beta}(r)$ for amorphous $\mathrm{GeSe}_{2}$ at the experimental density: experimental measurements from Ref. 21 (black lines) are compared to the results of FPMD calculations from Ref. 20 (dashed red lines) (with no account of the residual pressure) and to the results from Ref. 22 (green line, FPMD calculations accounting for the residual pressure).

It is of interest to analyze the sets of neighboring tetrahedron $\mathrm{Si}(1)-\mathrm{Si}(2)-\mathrm{Si}(1)$, since this triad is the most frequent when considering those forming chains connected in an edge-sharing fashion only. However, one finds that $\operatorname{Si}(1)$ and $\operatorname{Si}(0)$ centered triads are even more frequent. The highest value corresponds to $\mathrm{Si}(1)-\mathrm{Si}(0)-\mathrm{Si}(0)$ and $\mathrm{Si}(1)-\mathrm{Si}(1)-\mathrm{Si}(0)$, followed by $\mathrm{Si}(1)-\mathrm{Si}(1)-\mathrm{Si}(1)$ and 


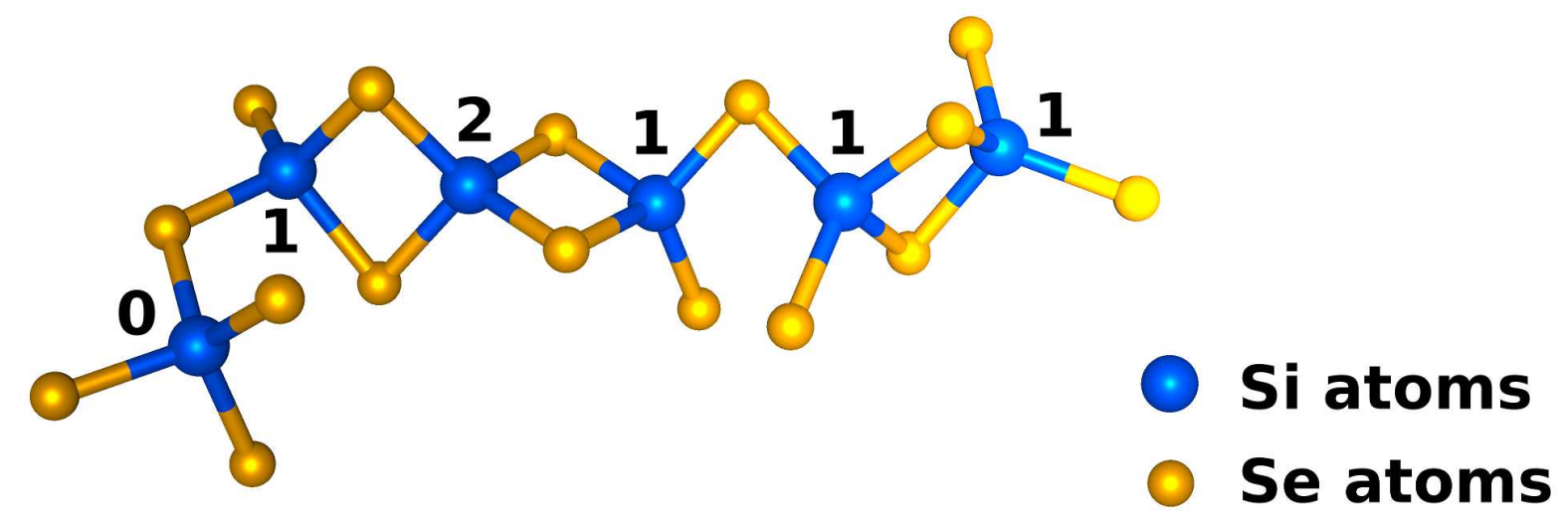

Figure 3: (Color online) Configurations showing the inter-tetrahedral connections resulting from sequences of $\mathrm{Si}$ atoms. Si atoms are labeled 2, 1, or 0 , according to their belonging to two $\mathrm{Si}(2)$, one $\mathrm{Si}(1)$, or zero $\mathrm{Si}(0)$ fourfold rings.

$\mathrm{Si}(1)-\mathrm{Si}(0)-\mathrm{Si}(1)$. These configurations involve corner-sharing and edge-sharing connections with coordination four (for $\mathrm{Si}$ ) and two (for $\mathrm{Se}$ ). Concerning the $\mathrm{Si}(2)$-centered motifs, $\mathrm{Si}(1)-\mathrm{Si}(2)-\mathrm{Si}(1)$ is the most abundant, while crystalline-like $\mathrm{Si}(2)-\mathrm{Si}(2)-\mathrm{Si}(2)$ chains could not be found. When counting the triads of tetrahedra within the network, we realized that the restriction to only one kind of inter-tetrahedral connection (the edge-sharing one) is by far not realistic, since both ES and CS occur in a non-negligible number of configurations. The set of results presented above is an outstanding example of the added value of the FPMD approach applied to chalcogenide glasses when one correctly considers them (as it should) as three dimensional structures, as opposed to methodologies (cluster calculations [24]) not accounting for the actual spatial extension of the network.

\section{Glassy $\mathrm{GeSe}_{4}$}

Glassy $\mathrm{GeSe}_{4}$ is representative of the structure of $\mathrm{Ge}_{\mathrm{x}} \mathrm{Se}_{1-\mathrm{x}}$ disordered networks in the Sepredominant range of compositions. For this composition, well beyond the stoichiometric one $(\mathrm{x}=0.33)$ on the Se side, the goal is to pinpoint the modes of connectivity among the $\mathrm{GeSe}_{4}$ tetrahedron and the Se atoms that cannot be bound to it. To this purpose, our calculations are focused on the relative percentage of Se-Se-Se, Ge-Se-Ge and Ge-Se-Se triads, referred to as BB, $\mathrm{AA}$ and $\mathrm{AB}$ units hereafter (Fig. 4). 


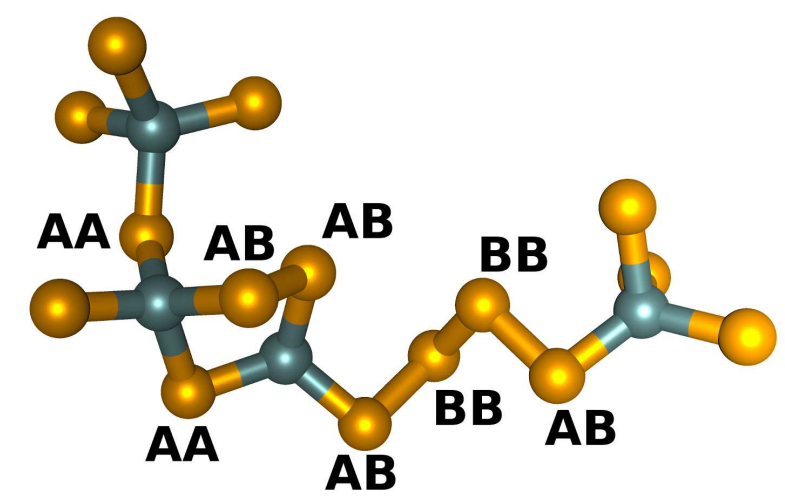

Figure 4: (Color online) $\mathrm{Ge}$ and Se atoms subunits taken from a FPMD model of $\mathrm{GeSe}_{4}$, in which $\mathrm{Ge}$ atoms are dark (grey) and Se atoms are light (orange). Se atoms located between two Se atoms are labeled BB, Se atoms between two Ge atoms are labeled AA, Se atoms between one Ge and one Se atom are labeled AB.

One can describe two opposite situations. In the first, the number of AB connections is very small, consisting with a phase separation between domains having as sub-compositions $\mathrm{GeSe}_{2}$ and $\mathrm{Se}_{n}$. This situation produces an essentially equal number of AA and BB units and very few (at the limit zero) $\mathrm{AB}$ units. In the second, Ge atoms are kept together via Se dimers (AB configurations). As a result, $\mathrm{AA}$ and $\mathrm{BB}$ configurations are lacking since residual Se atoms are not available to be part of chains longer than $\mathrm{Se}_{2}$ or to connect to nearest neighbors [25]. Therefore, in the case of glassy $\mathrm{GeSe}_{4}$ one would like to understand whether or not a phase separation (Se units separated from $\mathrm{GeSe}_{2}$ tetrahedra) can be really observed. As an additional goal to be attained from the methodological point of view, we are interested in the role played by the exchange-correlation functional in the context of our modelling choices for the density functional description of the electronic structure.

As a first step, we were able to consider the importance of the quenching procedure and of statistical fluctuations on the model created in the Perdew, Becke, Ernzerhof (PBE) [26] and in the Perdew-Wang (PW) [4] frameworks. We could establish that the differences between the PBE and the PW results is due to the different exchange-correlation recipes and cannot be ascribed to statistical fluctuations or to the quenching procedure. This means that some details of the short range structure in glassy $\mathrm{GeSe}_{4}$ are distinctly different in GGA-PW when compared to the GGAPBE scheme (for example, some minimal shifts in the positions of the peaks in $\mathrm{g}_{\mathrm{GeGe}}(r)$ as well as higher peaks and lower minima in the PBE case). In addition, the PBE scheme promotes very long 
$\mathrm{Se}_{n}$ chains $(n=12)$, by confirming the general description of a network structure featuring $\mathrm{GeSe}_{4}$ and $\mathrm{Se}_{n}$ units intimately connected. Based on the above pieces of evidence, two conclusions can be drawn on the atomic structure of glassy $\mathrm{GeSe}_{4}$. First, glassy $\mathrm{GeSe}_{4}$ is somewhat sensitive (and, in particular, the Se environment) to the kind of the bonding description (exchange-correlation functional) within density functional theory. Second, we could unambiguously affirm that there is no evidence on the modelling side for a phase separation, this results being demonstrated by the very high number of $\mathrm{AB}$ linkages.

\section{Glassy $\mathrm{GeS}_{2}$}

Our work on glassy $\mathrm{GeS}_{2}$ (see Ref. 27) was a benchmark FPMD calculation to be compared with molecular dynamics results obtained in the past via the non-self-consistent density functional Harris functional (HFMD) [28-32]. According to the HFMD description, glassy $\mathrm{GeS}_{2}$ contains a few homopolar bonds and threefold bonded sulfur atoms. It was of interest to perform a detailed comparison between the HFMD model and the FPMD model of bonding to obtain information on the similarities and differences between the two approaches. Being HFMD simpler to implement and yet much less expensive than FPMD, a favorable comparison between the two would have granted to HFMD the legitimacy of applications to other glassy structures. We have generated four uncorrelated FPMD trajectories for a model made of 480 atoms. For three trajectories the quench rates are very high, $\mathrm{q}(1)_{\mathrm{FPMD}}=5 \times 10^{14} \mathrm{~K} / \mathrm{s}$, while in the fourth case $\mathrm{q}(2)_{\mathrm{FPMD}}=3 \times$ $10^{13} \mathrm{~K} / \mathrm{s}$, the quench rate is more than ten times smaller. The resulting structural properties are very close, thereby proving that the dependence on the cooling rate cannot be taken as a crucial factor affecting the structure of the network, at least for the ranges of values compatible with the length of our trajectories. Despite a strong resemblance between the FPMD and HFMD sets of pair correlation functions, differences are found in the short-range environment of the Ge atoms, strongly tetrahedral within HFMD and highly defective in the FPMD case. By looking at the total neutron structure factor, $S(k)$, FPMD appears to improve the description of $g$-GeS 2 at intermediate range distances, as shown by the higher FSDP (figure 5).

One the purposes of this calculation was also to provide information on the bonding nature of $g-\mathrm{GeS}_{2}$ in comparison to the case of $g-\mathrm{GeSe}_{2}$ Overall, we have come to the conclusion that partial and total coordination numbers are not the adequate tools to gather any insight on specific bonding 


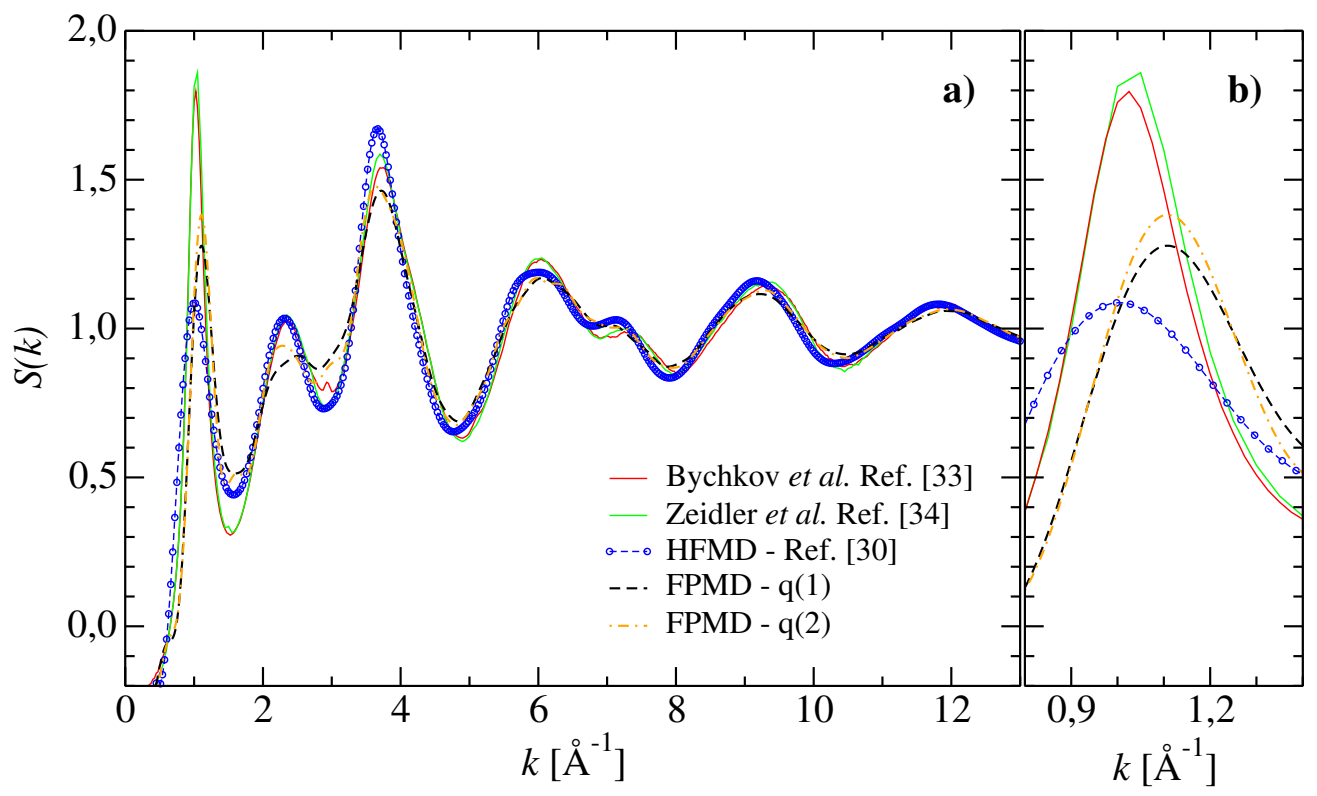

Figure 5: (Color online) a) Left: total neutron structure factor, $S(k)$, for $\mathrm{GeS}_{2}$. HFMD results from Ref. 30 (blue dashed line), experimental measurement by Bytchkov etal. Ref. 33 (red line) and by Zeidler etal. Ref. 34 (light green line), are compared to the FPMD results q(1) (black dashed line) and q(2) (orange dashed line). b) Right: zoom on the FSDP region of $S(k)$

characters differing in the two systems. Therefore, an electronic structure scheme based on the Wannier functions $[35,36]$ and centers has been exploited for a set of configurations pertaining to $g-\mathrm{GeS}_{2}$ and $g-\mathrm{GeSe}_{2}$. This approach was found particularly useful since it demonstrated that $g-\mathrm{GeSe}_{2}$ is less ionic than $g-\mathrm{GeS}_{2}$ in agreement with trends collected experimentally.

\section{Pressure effects}

A large research effort has been devoted to the study of structural changes in glassy networkforming disordered systems as a result of pressure effects. These investigations have been carried out in close interaction with the experimental group of P.S. Salmon in Bath, performing in situ high pressure neutron diffraction experiments. In these experiments, the isotope substitution method is employed to understand the correlations hidden in a single diffraction pattern.

In the case of glassy $\mathrm{GeSe}_{2}[37$ ] and for the range from ambient pressure to $\mathrm{P} \simeq 8.5 \mathrm{GPa}$ the neutron diffraction results are in very good agreement with the outcome of FPMD calculations. The comparison is very favorable for difference functions (Fig. 6), the mean nearest-neighbor 
bond distance $\bar{r}$, the coordination number $\bar{n}$, and the Se-Ge-Se bond angle (Fig. 7).
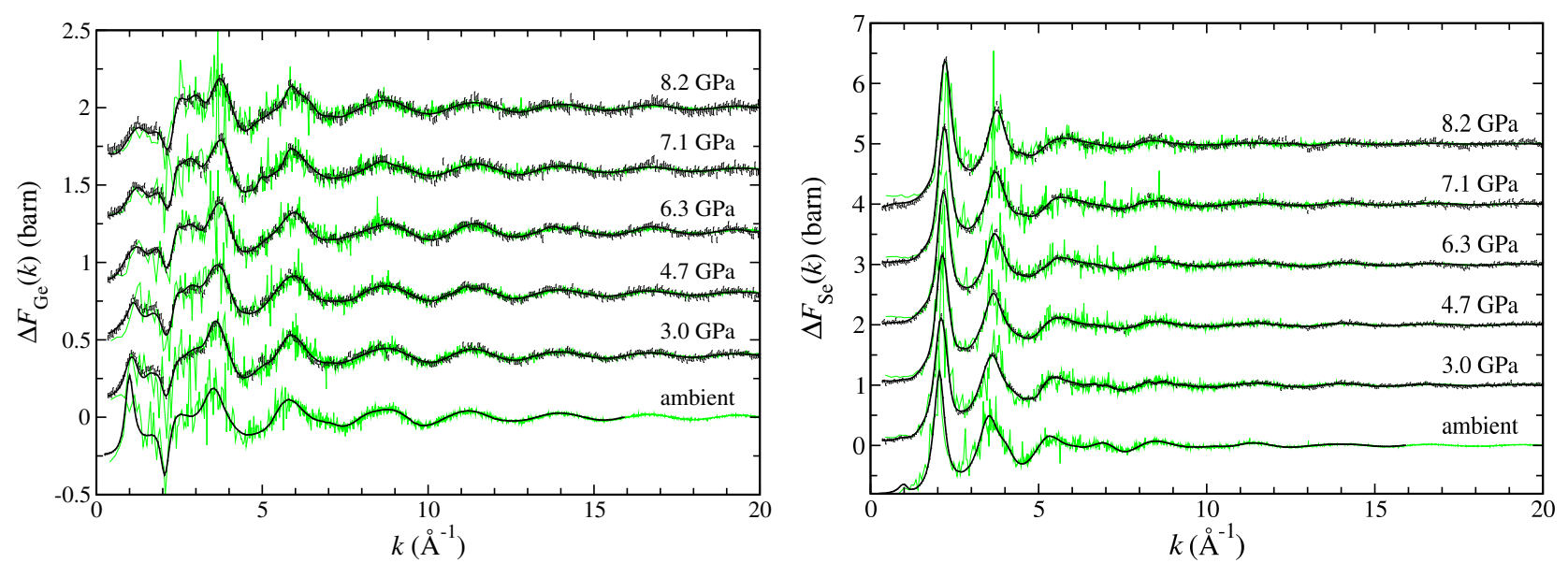

Figure 6: (Color online) The difference functions $\mathrm{DF}_{\mathrm{Ge}}(k)$ (barn) $=0.236(4)\left[S_{\mathrm{GeSe}}(k)-1\right]+$ $0.099(2)\left[S_{\mathrm{GeGe}}(k)-1\right]\left(\right.$ left panel) and $\mathrm{DF}_{\mathrm{Se}}(k)($ barn $)=0.184(3)\left[S_{\mathrm{GeSe}}(k)-1\right]+0.588(11)\left[S_{\mathrm{SeSe}}(k)-1\right]$ (right panel) as measured with the diffractometer D4 at ambient pressure (Ref. 21) and at pressures of 3.0(5), 4.7(5), 6.3(5), 7.1(5), and 8.2(5) GPa (vertical error bars on data points). Comparison is carried out with FPMD results at ambient pressure and at 3.4, 4.88, 7.25, 8.73, and 9.87 GPa, light (green) curves. The dark (black) curves are the back Fourier transforms of the r-space difference functions obtained via measurements.

The changes in the structure arising from an increase of the density in $\mathrm{GeSe}_{2}$ are very much different from those occurring in oxide glasses such as $\mathrm{GeO}_{2}, \mathrm{~B}_{2} \mathrm{O}_{3}$ and $\mathrm{SiO}_{2}$. The case of $\mathrm{GeSe}_{2}$ features a large number of edge-sharing connections, while in oxide glasses corner-sharing connections are predominant over the range of densities for which the coordination numbers are the same as those of ambient-pressure conditions [38-44].

However, when the pressure increases, there is a noticeable divergence between experiments (diffraction results) and FPMD data, since the diffraction results exhibit a moderate increase in $\bar{r}$ and $\bar{n}$. Upon increasing the density, the FPMD model is characterized by the existence of fivefold and sixfold coordinated Ge atoms. These are responsible for the formation of several ES-made motifs, together with Se atoms threefold, fourfold, and fivefold coordinated. Interestingly, many of these highly coordinated Se and Ge atoms are found to form homopolar bonds. Therefore, one can conclude that homopolar bonds play a major role in driving the structural transformation at high densities. The opposite occurs in oxides for which at all pressures one observes the 


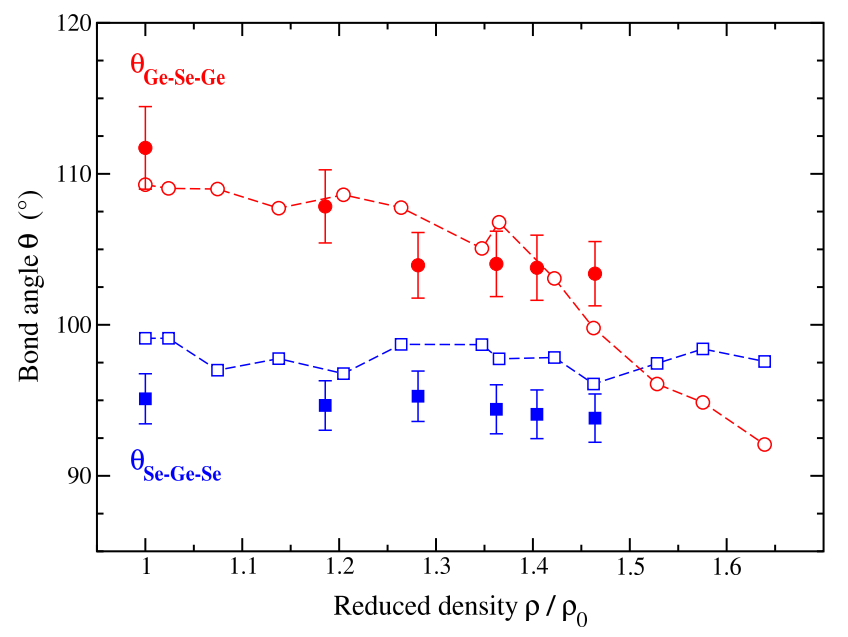

Figure 7: (Color online) The $\rho / \rho_{0}$ dependence of the mean intra-polyhedral Se-Ge-Se (red $\bullet$ ) and interpolyhedral Ge-Se-Ge (blue $\mathbf{0}$ ) bond angles as estimated from the measured Ge-Se, Ge-Ge and Se-Se distances. The experimental results are compared to the mean values $\left\langle\theta_{\mathrm{SeGeSe}}\right\rangle$ (broken red curve with $\bigcirc$ symbols) and $\left\langle\theta_{\mathrm{GeSeGe}}\right\rangle$ (broken blue curve with $\square$ symbols) obtained from FPMD.

persistence of chemical order [38-43]. A comment is in order concerning the different behavior of the neutron diffraction data and the first-principles model. This can be ascribed to the presence of a barrier to be surmounted allowing for substantial structural changes. Due to this energy barrier, such changes cannot be observed in compression experiments carried out at room temperature. However, the opposite can occur when higher temperatures are attained in simulations. More specifically, having identified one valley in the energy surface for pressures up to $\sim 8.5 \mathrm{GPa}$ $\left(\rho / \rho_{0} \simeq 1.42\right)$, it is realistic to postulate the existence of two possible branches for larger pressures leading to two valleys kept apart by an energy barrier readily overcome at temperatures of the order of $900 \mathrm{~K}$. Overall, we have observed an enhanced stability of ES tetrahedral upon densification, in line with the fragility of liquids prone to form glasses [45]. In addition our study has highlighted the impact of homopolar bonds in promoting the structural change toward higher-coordinated polyhedral.

The structure of glassy $\mathrm{GeSe}_{4}$ at pressures up to $\sim 14.4 \mathrm{GPa}$ was also considered by employing both neutron diffraction and FPMD [46]. First, it appears that the neutron data agree well with x-ray diffraction results [47] by showing that there are no important modifications in the mean coordination number $\bar{n}$ for pressures smaller than 8.6 GPa. FPMD is consistent with the idea 
that the chemical and topological order are essentially unaltered in glassy $\mathrm{GeSe}_{4}$ over the range of pressures up to $\sim 8 \mathrm{GPa}$. On the one hand, edge-sharing $\mathrm{Ge}\left(\mathrm{Se} \frac{1}{2}\right)_{4}$ tetrahedral motifs have a high degree of rigidity. On the other hand, $\mathrm{Se}_{n}$ chains act within the network to ensure enhanced flexibility. For these reasons, the main structural motifs are preserved throughout densification.

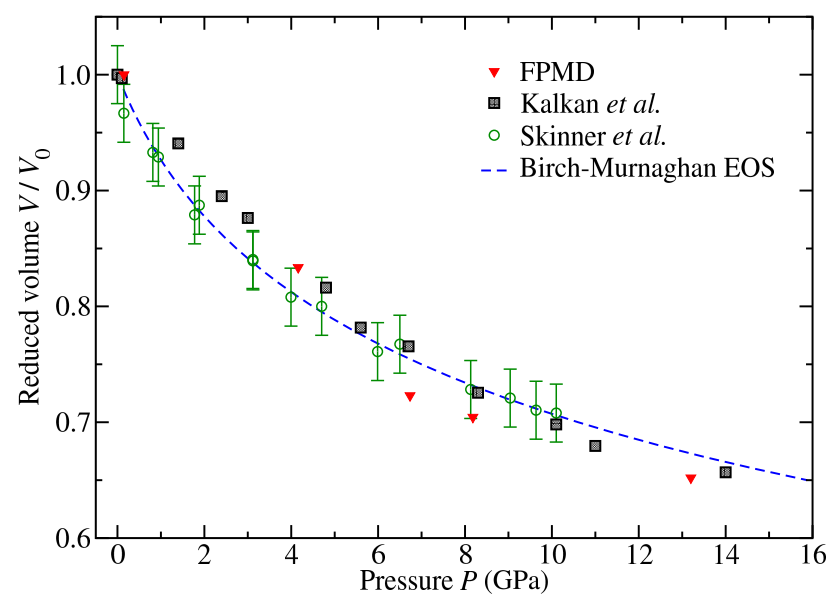

Figure 8: (Color online) Equation of state pressure/volume for glassy $\mathrm{GeSe}_{4}$ obtained via FPMD calculation (red up and down triangle). Experimental data under compression are provided as measured by Skinner et al. [47], and Kalkan et al. [48], green circles with error bars and filled black squares, respectively. We also present the third-order Birch-Murnaghan fit of experimental data from Ref. 47(blue dashes).

The mean coordination number $\bar{n} \simeq 2.4$ reflect the nature of the network rearrangement and confirms the role played by the $\mathrm{Se}_{n}$ chains. For pressures around $13 \mathrm{GPa}$, a decrease by $\simeq 15 \%$ in the number of $\mathrm{Ge}\left(\mathrm{Se} \frac{1}{2}\right)_{4}$ tetrahedral is recorded. Indeed, 5-fold coordinated Ge atoms appear together with homopolar Ge-Ge bonds. Also, 3-fold coordinated Se atoms are more numerous. The effect of pressure can also be analyzed on the basis of electronic properties. For instance, mid-gap states are visible beginning at a pressure $6.73 \mathrm{GPa}$. These states are due to Se atoms one fold coordinated at the end of $\mathrm{Se}_{n}$ chains, since the pressure is able to break Se-Se homopolar bonds being part of $\mathrm{Se}_{n}$ chains.

\section{Ternary systems}

More recently (see Ref. 49), we have devoted interest to chalcogenide ternary systems that are relevant for applications in the area of phase change materials [50]. Some chalcogenide alloys 
undergo a very rapid and reversible transformation between the crystalline and the amorphous phases when undergoing a thermal treatment. The materials that can be described as bearing such property fall in the category of phase change materials (PCMs), largely used in electronic memory devices and disks memories. It is possible to differentiate the two memory states of PCMs since the crystalline phase and the disordered phase of the chalcogenide have quite distinct optical and electrical resistivity. We have selected $\mathrm{Ga}_{4} \mathrm{Sb}_{6} \mathrm{Te}_{3}$ since it features enhanced high electrical contrast, speed of crystallization, and crystallization temperature, this factor being of great importance for applications at high temperatures. In particular, we have focused on the structural properties with the intent of providing information on the basic units discernible within the network. Our models for the amorphous phase were created via the procedure of quenching from the liquid state at fixed density. The sizes of the systems were taken to correspond to 117 and 299 atoms. When looking at the different bond identities, we detected a very small number of Sb-Te bonds. Overall, amorphous $\mathrm{Ga}_{4} \mathrm{Sb}_{6} \mathrm{Te}_{3}$ is a blend of GaTe and GaSb networks, mostly containing tetrahedra and coexisting with a sizeable fraction of $\mathrm{Sb}$ atoms. If it remains true that a large majority of $\mathrm{Ga}$ atoms are in tetrahedral short-range linkages, we also found Ga-Ga dimers. These are reminiscent of crystalline GaTe. The case of antimony is quite different. We have two distinct environments, i.e. for threefold coordinated atoms the pyramidal motif prevails, while for fourfold coordinated atoms the tetrahedral one is dominant. One observes an important concentration of Sb when compared to GaSb-GaTe. This results in an incomplete and yet quite substantial segregation of antimony, leading to clusters having a topological organization reminiscent of the one of crystalline $\mathrm{Sb}$ (see figure 9).

These structural details are very close to those found in amorphous $\operatorname{In}_{3} \mathrm{SbTe}_{2}$ [51] which exhibits very few Sb-Te bonds and local structures bearing resemblance to those present in InTe and InSb.

\section{The structure of glassy $\mathrm{GeTe}_{4}$ and the role of dispersion forces}

Interest in amorphous $\mathrm{GeTe}_{4}\left(a-\mathrm{GeTe}_{4}\right)$ is motivated by its intrinsic thermal and optical properties $[52,53]$ and potential applications in memory cells. $[54,55]$ When referring to phase-change alloys, one realizes that $\mathrm{GeTe}_{4}$ is quite recurrent in ternary systems including Ge and $\mathrm{Te}$ in their compositions. However, the structure of $\mathrm{Ge}_{x} \mathrm{Te}_{1-x}$ at concentrations $x \sim 0.2$ remains elusive. There are also other reasons that motivate first-principles modelling on this glass. Previous re- 


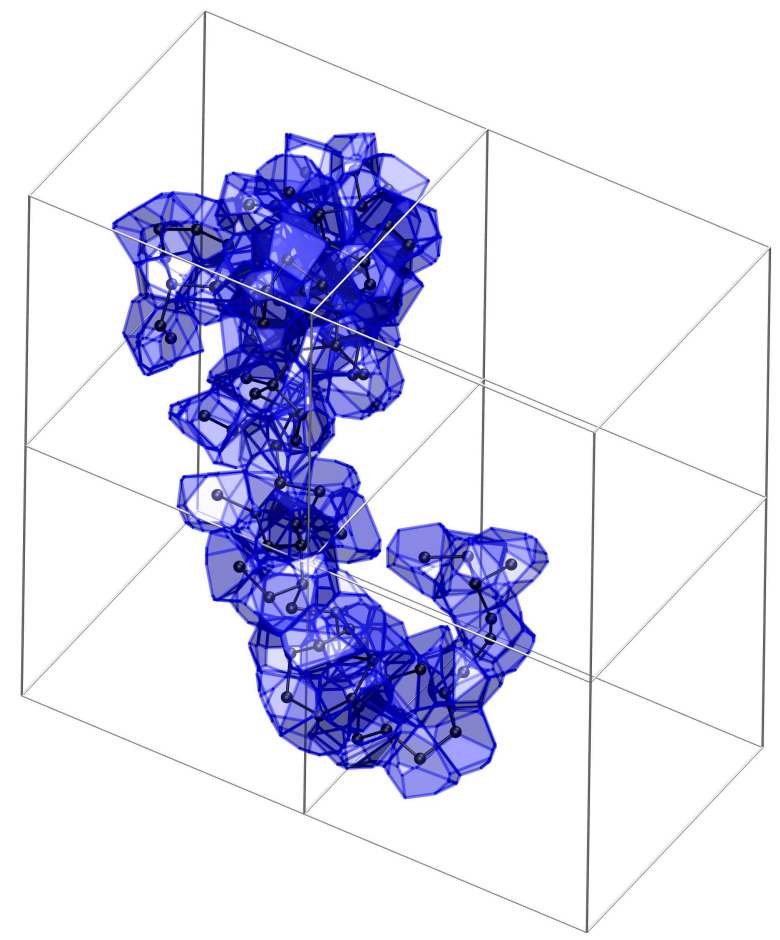

Figure 9: (Color online) Illustration of the clustering of $\mathrm{Sb}$ atoms in amorphous $\mathrm{Ga}_{4} \mathrm{Sb}_{6} \mathrm{Te}_{3}$. The model is 299 atoms large, the atoms of the cluster are show in dark (black), the shape of the cluster is highlighted in light translucent color (blue) of the Voronoï polyhedra built around the Sb atoms. A $2 \times 2$ supercell is shown with no application of periodic boundary so as to highlight both shape and connectivity of the cluster.

sults suggested that substantial improvements in the structure factor and in the pair correlation functions of liquid $\mathrm{Ge}_{15} \mathrm{Te}_{85}$ can be achieved when the van der Waals (vdW) dispersion forces are accounted for. [56] It should be underlined that, in what follows and in the context of the study of glassy $\mathrm{GeTe}_{4}$ [57], the dispersion forces had been first been considered by employing the Grimme [58] recipe. This is not the only methodology available for including the vdW contributions. In the second part of this section, (devoted to the test case of liquid $\mathrm{GeSe}_{2}$ and to the most recent calculations on glassy $\mathrm{GeTe}_{4}$ ), we shall come back to the predictive power of the Grimme recipe when compared to other schemes better rooted within the electronic structure of a given system. As mentioned before in this review and in particularly for $\mathrm{GeSe}_{2}$, the $\mathrm{XC}$ functional did play a major role in determining the basic structural features. This was made explicit by the description of the short-range Ge environment resulting from the adoption of the Becke, Lee, Yang, and Parr (BLYP) recipe [59-61]. This scheme is capable of attenuating the delocalized behavior of 
the electron density that favors the metallic character by preventing from a correct description of iono-covalent bonding effects.

In view of these considerations we studied the structural properties of glassy $\mathrm{GeTe}_{4}$ by employing two different exchange correlation functionals and accounting or not for the vdW dispersion forces. [57] Accordingly, we have modelled glassy $\mathrm{GeTe}_{4}$ with the XC functional PBE (PBE hereafter), [26] as XC functional and the presence or the absence of vdW forces, the same combinations being used for the BLYP XC functional. For each model, we have targeted the total pair correlation function $g(r)$ and the total structure factor $S(k)$ given in figure 10, as well as additional structural properties (bond angle distributions, partial correlation function, local order parameters and coordination numbers).
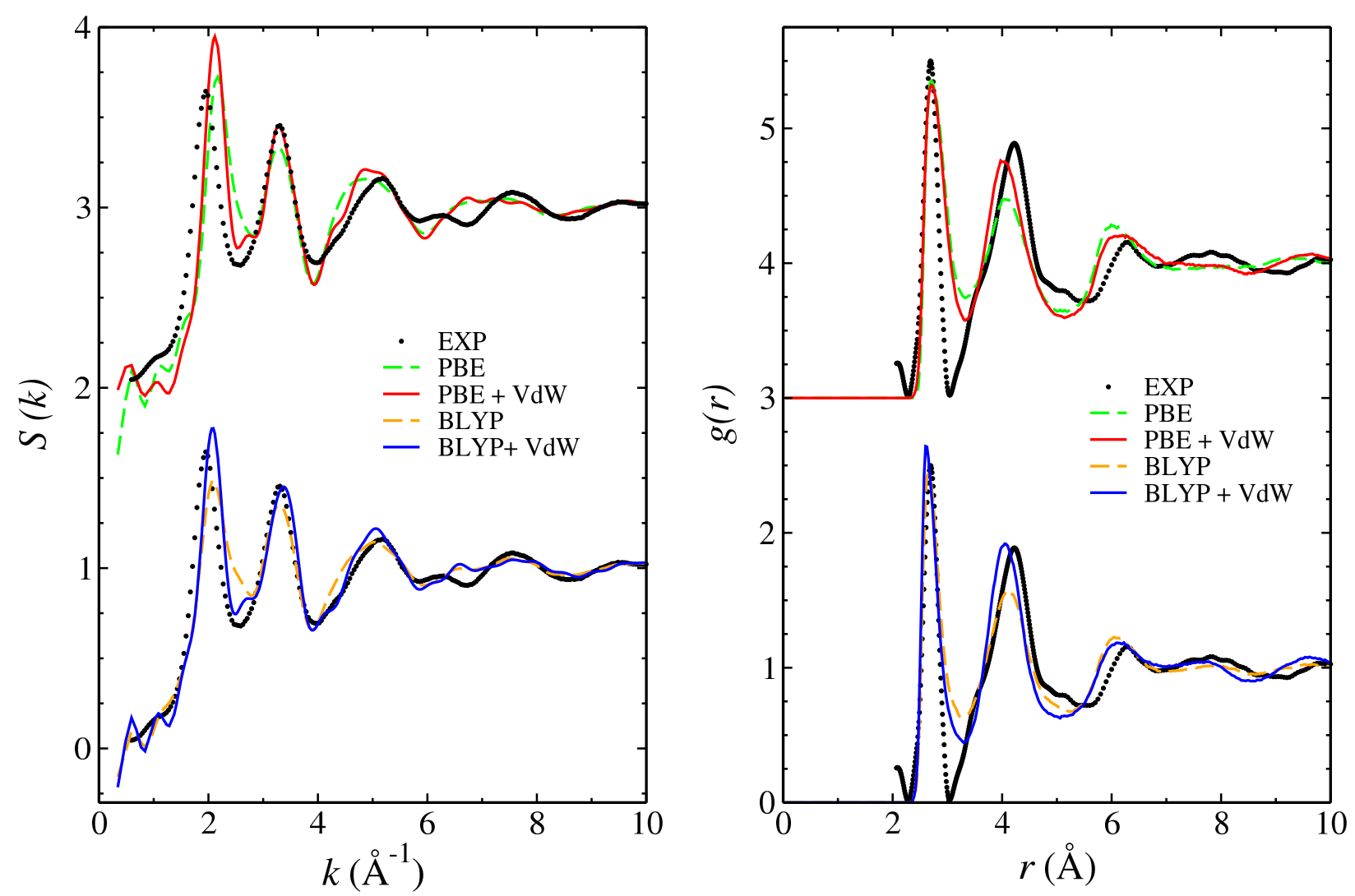

Figure 10: (Color online) Total structure factor $S(k)$ (left panel) and pair correlation function (right panel) for $\mathrm{GeTe}_{4}$. FPMD models calculated using the BLYP functional (orange dashes) and the PBE (light green dashes) functional, as well as the BLYP + vdW (blue line) and PBE + vdW (red line) from Ref. 57, are shown in comparison with experimental data from Ref. 62. 
Focusing on the issue of the vdW contribution, it appears that its inclusion improves the total pair correlation function and structure factor in the PBE and (to a smaller extent) BLYP cases (see figure 10). The best agreement with the experimental data is found when applying BLYP used in combination with the vdW forces. The corresponding network topology features the predominance of the tetrahedral motifs coexisting with some coordination defects. PBE appears to favor instead a coexistence of octahedral and tetrahedral units. Therefore, it is important to account for dispersion forces to properly describe Ge-Te chemical bonding in disordered systems. In particular, we found that the use of BLYP as exchange-correlation recipe is instrumental to achieve an optimal reproduction of available experimental properties. The resulting atomic structure is made of a large majority of tetrahedra coexisting with a small number of defects, thereby differing from previous predictions based on comparable percentages of tetrahedral and octahedral coordination structures

Quite recently we have performed a comparative test of the performances of the vdW scheme due to the Grimme [58] and an alternative methodology derived from the formalism of the maximally localized Wannier functions (MLWF) [63]. The selected system is liquid $\mathrm{GeSe}_{2}\left(l-\mathrm{GeSe}_{2}\right)$ for which FPMD data have already been obtained without accounting for dispersion forces. [15] We found useful to mention the most important results of this analysis within this review paper, since the account of dispersion forces is becoming more and more ubiquitous in density functional approaches. The scheme due to Grimme contains a coefficient related to the intensity of the attractive forces (long range). By construction, this coefficient is not bound to evolve as a function of the evolution in time of the electronic structure. This occurs instead in the scheme inspired by the formalism of the maximally localized Wannier functions (MLWF) [63]. Having recalled the basic difference between the two strategies to account for the dispersion forces, we found that the topology of the $l$-GeSe $\mathrm{G}_{2}$ network was strongly insensitive to the presence or the absence of the dispersion forces. [64] In particular, the MLWF scheme and the pristine FPMD approach were found to lie very close in terms of structural data. A surprising effect was observed when using the Grimme approach. Indeed, the partial pair correlation functions (see figure 11) and the Ge-Se-Ge bond angle distribution did feature some additional contribution, in apparent disagreement with the MLWF and no vdW FPMD [64].

When comparing the corresponding networks, the MLWF approach favors the corner-sharing connections over the edge-sharing ones. Therefore, it looks like the different treatments of the vdW 

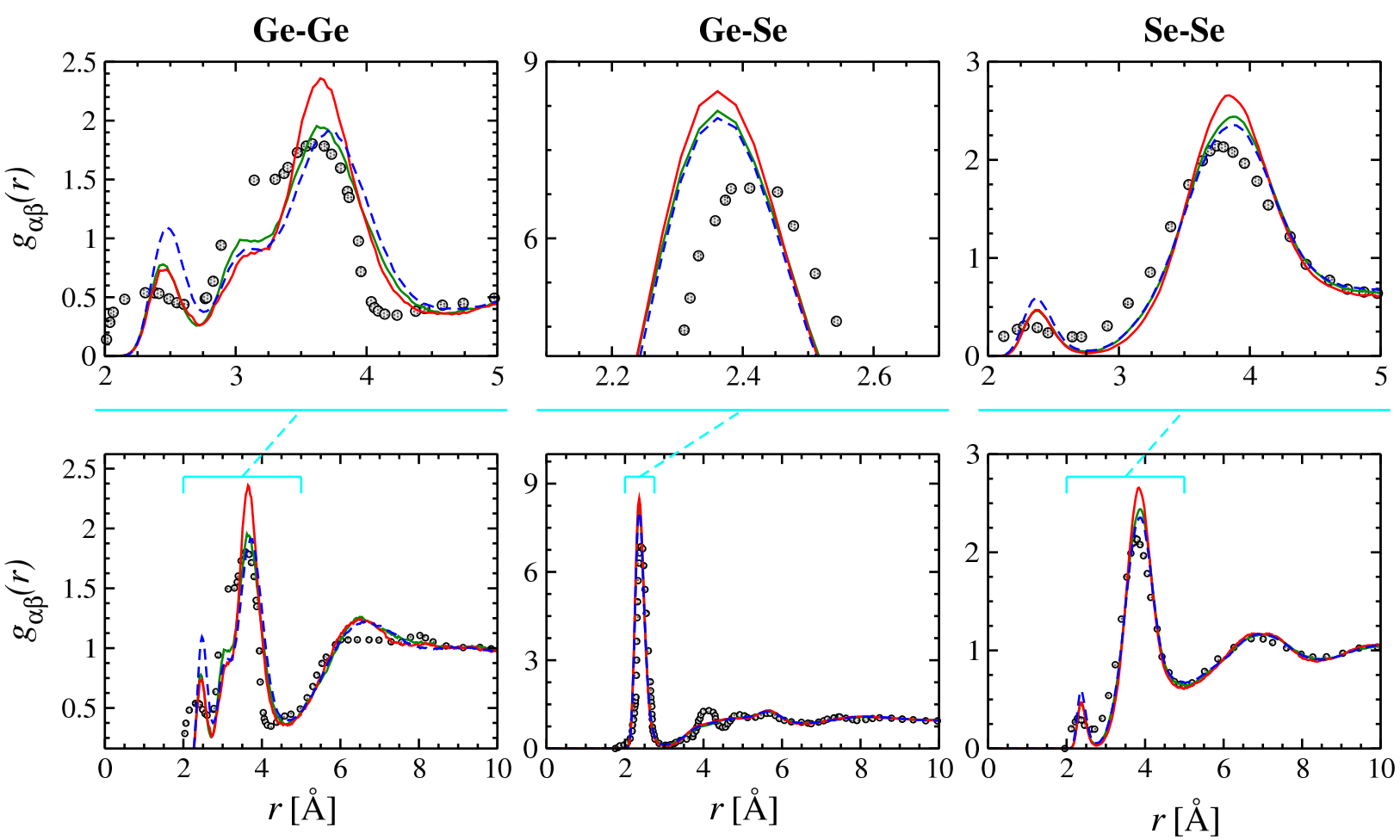

Figure 11: (Color online) Partial pair correlation functions for $l$-GeSe $e_{2}$ from Ref. 64. The results obtained with the different FPMD approaches to treat (or not) van der Waals interactions, BLYP without vdW (green line), BLYP + Grimme DFT-D2 (red line), BLYP + Wannier MLWF (blue line) are compared to their experimental counterparts from Ref. 16 (dots). Bottom panel: global overview. Top panel: zoom-in on first and second peak regions.

contribution are by far not behaving in a unique manner, the less empirical recipe (MLWF for instance) being able to minimize the impact of the dispersion forces when they are expected to play only a minor role. These conclusions have been substantiated by very recent work carried out on glassy $\mathrm{GeTe}_{4}[65]$.

In that paper, it appeared that updating the vdW contribution (as in the Wannier approach) as a result of modifications in the electronic structure occurring during the temporal trajectory led to results dissimilar from those issued by the Grimme-D2 framework. Interestingly, the Wannier strategy is characterized by the production of structural features agreeing well with those calculated when there is no account of dispersion forces. Therefore, it turns out that the Wannier and the Grimme recipes for the dispersion forces are somewhat quite different in terms of a small and yet not negligible set of structural properties (figure 12). It remains to be understood whether the 


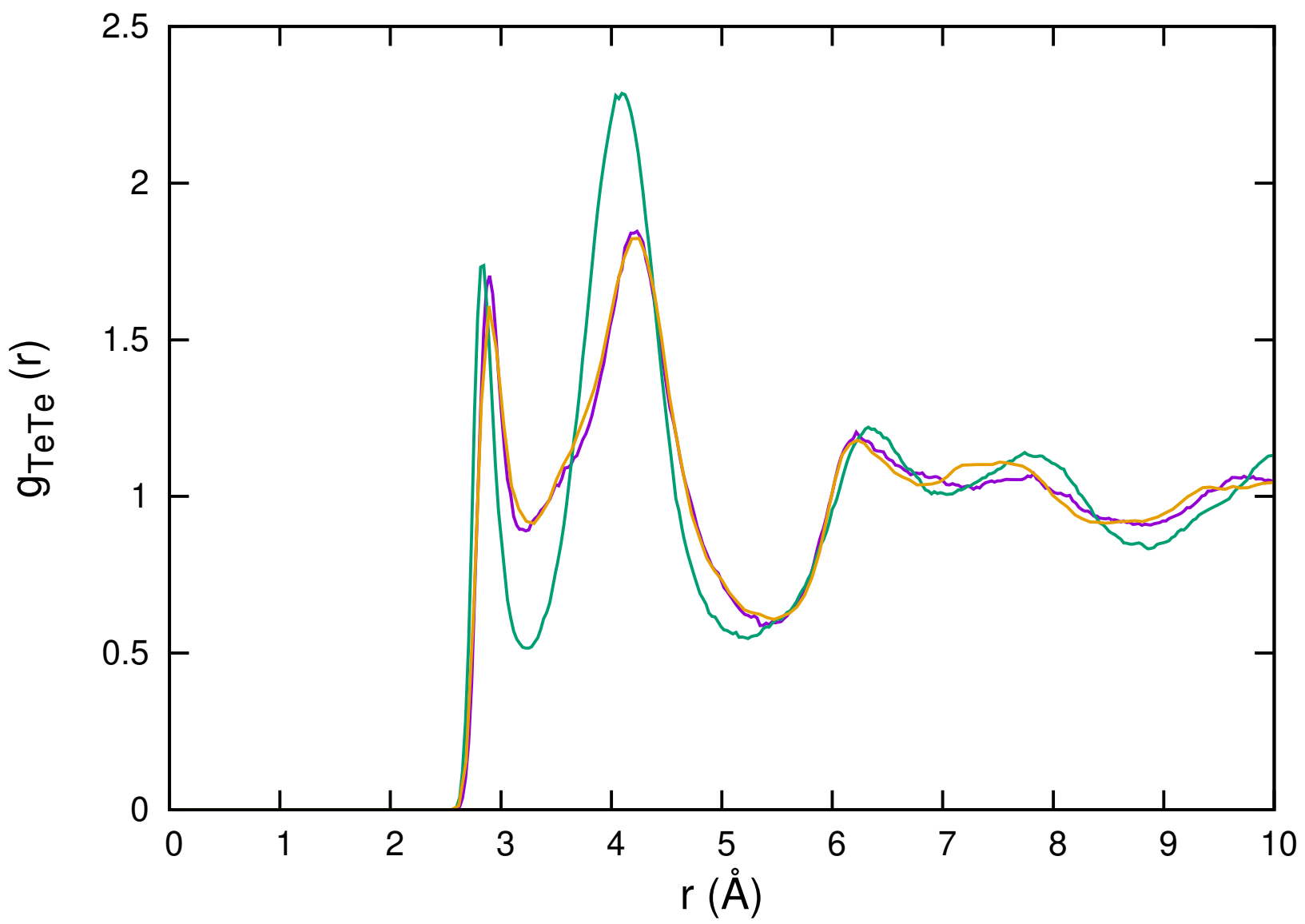

Figure 12: (Color online) Behavior of the pair correlation function Te-Te of glassy $\mathrm{GeTe}_{4}$. Three results are reported: without considering the dispersion forces (purple line), by using the Grimme-D2 scheme (green line) and by using the MLWF scheme for the dispersion forces (orange line).

better agreement with experiments featured by the Grimme-D2 scheme has some real foundations or it is somewhat fortuitous. Also, it is desirable that all recipes for the dispersion forces could play a negligible role when their impact is expected to be smaller in comparison to other bonding contributions. This is not exactly what happens in the cases treated via Grimme-D2, as exemplified in our recent calculations.

FPMD vs. Classical potential for disordered network forming materials: the case of liquid $\mathrm{GeSe}_{2}$

There is a longstanding quest for atomic-scale methods built on the electronic structure and yet prone to be formulated in affordable analytical forms. Along these lines, we selected $l$-GeSe 2 as a test case for the construction of a suitable interatomic potential [66]. With the purpose of 
constructing interatomic potentials working realistically, it is appropriate to take advantage of the notion of ionicity by attributing a charge to each atom. The resulting interatomic potentials contain a two-body part, combined with a description of many-body ionic polarization in addition to the predominant Coulombic interaction. It is of interest to ascertain whether models constructed along these criteria can be useful to gather information on the structure of $l$-GeSe $\mathrm{Ge}_{2}$ It should be reminded that models employing formal charges and known as rigid-ion models are quite satisfactory in accounting for the static structures of $\mathrm{SiO}_{2}$ and $\mathrm{BeF}_{2}$ but are much less performing for $\mathrm{ZnCl}_{2}, \mathrm{GeSe}_{2}$ or $\mathrm{BeCl}_{2}$. This is due to the different values (acute or obtuse) taken by the angles formed by triads of atoms within the system. To improve the performances of these models, the effective potential can be enriched by including a many-body polarization term, as done in the context of the polarizable-ion model, PIM. [67] A PIM for $l-\mathrm{GeSe}_{2}$ was devised by Sharma and Wilson. [68] This model overestimates $\left(T_{m}=2900 \mathrm{~K}\right)$ the system melting point (experimental value of $T_{m}=1025 \mathrm{~K}$ ) and does not allow for the occurrence of homopolar bonds. Despite its intrinsic limitations, the potential is quite effective when it comes to describing intermediate range order, being well adapted to be the ionic model counterpart of the FPMD modelling of liquid $\mathrm{GeSe}_{2}$. Given these considerations, we carried out a comparison between the FPMD and the PIM models for $l-\mathrm{GeSe}_{2}$, by obtaining two distinct structures for $l-\mathrm{GeSe}_{2}$ [66]. In what follows, we report some conclusions of that work, intended to highlight the intrinsic limitations of the PIM effective potential scheme when compared to FPMD. The network obtained by using FPMD is a predominant collection of tetrahedra coexisting with a non-negligible quantity of homopolar bonds and miscoordinations. The low temperature PIM model instead is highly chemically ordered, homopolar bonds being absent and Ge atoms coordinated other than in a fourfold manner being only a few. When the temperature is increased, PIM and FPMD structures become similar. However, the absence of homopolar bonds persists in the PIM scheme. Therefore, it remains true that the PIM potential cannot be taken as a fully reliable model of $l$-GeSe $e_{2}$, thereby confirming the need of quantitative, first-principles tools to describe chalcogenide disordered materials. This statement can be further substantiated by observing that within the PIM approach the experimental diffusion coefficient recorded at $T=1050 \mathrm{~K}$ can be recovered at the quite unrealistic temperature of $T=7000 \mathrm{~K}$. 


\section{Thermal conductivity of glassy $\mathrm{GeTe}_{4}$}

By targeting the thermal conductivity, FPMD can help to elucidate the microscopic origin of heat transport processes. To this purpose, and for the first time in the case of FPMD studies, molecular dynamics has been combined to a new methodology, termed AEMD (approach-to-equilibrium molecular dynamics, [69]) in order to obtain the thermal conductivity of glassy $\mathrm{GeTe}_{4}$. AEMD is based on the establishment and analysis of thermal transients, without requiring a stationary state to be reached. In fact, all information allowing for the description of the thermal event is available within the time of decay of the transitory regime. This allows for a gain of one order of magnitude in computational time with respect to other molecular dynamics approaches. We recall that the AEMD method consists in imposing a temperature difference between two blocks in which the system is divided (phase 1). This is followed by a release of such local thermal constraint so as to induce a transient regime (phase 2), allowing for the temperature of the hot block to decrease and the temperature of the cold block to increase. Figure 13 shows that this entire process is within the reach of the time scale accessible to FPMD, at least for the case of $\mathrm{GeTe}_{4}$.

The difference of temperature between the hot block and the cold block follows an exponential decay during phase 2 , reminiscent of the heat conduction problem for a ring [70]. This similarity provides a route to relate the decay time to the thermal conductivity of the material. We obtain a thermal conductivity equal to $0.02 \pm 0.01 \mathrm{~W} \mathrm{~m}^{-1} \mathrm{~K}^{-1}$ at $130 \mathrm{~K}$ for a system containing 185 atoms. The experimental value resulting from using a parallel temperature conductance technique is 0.14 $\mathrm{W} \mathrm{m}{ }^{-1} \mathrm{~K}^{-1}$ [71]. This technique leads to an overestimate by $20-25 \%$ of the thermal conductivity when compared to a measurement "laser flash" [71]. This last experimental framework is closer to our simulation methodology. As a consequence, we find more appropriate to refer to an experimental value for the thermal conductivity of $\approx 0.1 \mathrm{~W} \mathrm{~m}^{-1} \mathrm{~K}^{-1}$. The above underestimation of the thermal conductivity obtained by AEMD is due to size effects that have a physical origin. The sensitivity of AEMD to the system size is intimately related to the existence of extended free paths of the heat carriers [72]. The latest are expected to be very limited in the present case due to the disordered nature of the system. However, in order to test this hypothesis, we have performed a second set of calculations with 370 atoms (dimension doubled in the direction of heat transfer). The thermal conductivity is increased by more than a factor 2 to $\approx 0.05 \mathrm{~W} \mathrm{~m}^{-1} \mathrm{~K}^{-1}$, by approaching the experimental data. Therefore, the observed size dependence highlights the existence of 


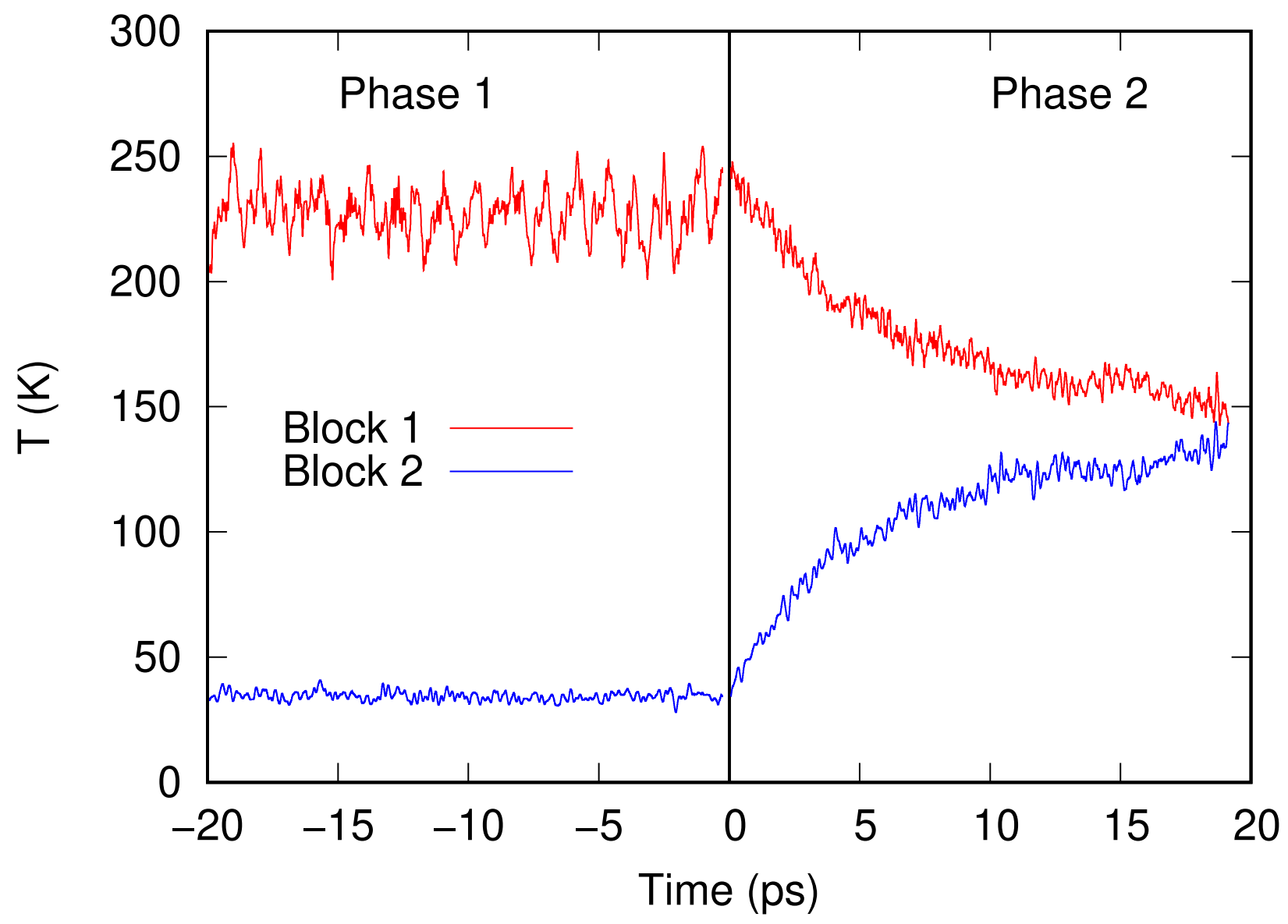

Figure 13: (Color online) Time evolution of the temperature of the blocks during the two AEMD phases.

propagative modes of heat transport on distances well above the range of structural order in the material.

A DETAILED EXAMPLE OF A STUDY WITHIN FPMD: THE CASES OF GE $\mathrm{SE}_{3}$ AND GESE $_{9}$

\section{Introduction}

The following sections of this chapter will present and compare results obtained for glassy $\mathrm{Ge}_{2} \mathrm{Se}_{3}$ and glassy $\mathrm{GeSe}_{9}$ These two glasses are positioned close to the opposite sides of the composition range of the $\mathrm{Ge}_{\mathrm{x}} \mathrm{Se}_{1-\mathrm{x}}$ family. The $\mathrm{Ge}_{2} \mathrm{Se}_{3}$ system, with $x=0.4$, is the one with the highest concentration of Ge atoms having the ability to form a glass after quenching. Conversely, the $\mathrm{GeSe}_{9}$ system, with $x=0.1$, is very close to the concentration threshold required to detect the intermediate range order in the amorphous phase and the first sharp diffraction peak in the structure factor. The comparison of these two systems is an instructive way to capture more extensively 
the chemical and the physical properties of $\mathrm{Ge}_{\mathrm{x}} \mathrm{Se}_{1-\mathrm{x}}$ glasses.

\section{Theoretical Models}

$\mathrm{Ge}_{2} \mathrm{Se}_{3}$

To perform the simulations we selected a system with $N=120$ (48 $\mathrm{Ge}$ and $72 \mathrm{Se}$ ) atoms in a periodic cubic cell of size $15.21 \AA$, at the experimental density of the glass at $300 \mathrm{~K}$, $\rho_{\text {exp }}=4.3271 \mathrm{~g} / \mathrm{cm}^{3}$ [73]. For the description of the electronic structure within DFT we selected as generalized gradient approximation (GGA) the form due to Becke (B) for the exchange energy and Lee, Yang and Parr (LYP) for the correlation energy [6, 7]. Car and Parrinello FPMD has been employed throughout [74]. Valence electrons were treated in conjunction with norm conserving Trouiller-Martins pseudopotentials [75]. The expansion of the wave functions was carried out at the $\Gamma$ point of the supercell with an energy cutoff $E_{c}=20$ Ry for the plane waves basis set. The fictitious electron mass is 2000 a.u. (i.e. in units of $m_{e} a_{0}^{2}$ where $m_{e}$ is the electron mass and $a_{0}$ is the Bohr radius) and the time step for the integration of the Newton dynamics is $\Delta t=0.24 \mathrm{fs}$. Temperature control follows the Nosé-Hoover thermostats scheme[76-78].

Ten initial configurations separated by 10 ps were taken from our previous work on liquid $\mathrm{Ge}_{2} \mathrm{Se}_{3}[79]$. For each one of them, the protocol to quench the $\mathrm{Ge}_{2} \mathrm{Se}_{3}$ liquid goes as follows: (a) the density of the liquid configuration at $T=1000 \mathrm{~K}$ was modified to take the value of the glass [73], (b) the $\mathrm{Ge}_{2} \mathrm{Se}_{3}$ system was equilibrated at $T=1000 \mathrm{~K}$ for $10 \mathrm{ps}$, and (c) the temperature of the thermostat was set to $T=900 \mathrm{~K}, T=600 \mathrm{~K}$ and $T=300 \mathrm{~K}$, with annealing over $20 \mathrm{ps,}$ 30 ps and 50 ps, respectively. Statistical averages were calculated over each sub-trajectory along a total time interval of 52 ps at $T=300 \mathrm{~K}$. Results were obtained by taking the mean over the sets of data collected on the ten sub-trajectories.

$\mathrm{GeSe}_{9}$

Calculations were performed on $N=260(26 \mathrm{Ge}$ and $234 \mathrm{Se})$ atoms in a periodic cubic cell of size $19.9 \AA$ at the density of the glass as given by the experiments at $300 \mathrm{~K}, \rho_{\text {exp }}=4.29 \mathrm{~g} / \mathrm{cm}^{3}$ [80]. The system size ensures the access to a minimum magnitude of the scattering vector $k_{\min }=$ $0.3157 \AA^{-1}$, much smaller than the location of the FSDP at $k_{\mathrm{FSDP}} \simeq 1 \AA^{-1}$ for $\mathrm{Ge}_{\mathrm{x}} \mathrm{Se}_{1-\mathrm{x}}$ glasses [73]. The electronic structure calculation method follows the same guidelines as in the case of 
glassy $\mathrm{Ge}_{2} \mathrm{Se}_{3}$ reported above. In Ref. 80 several models with different thermal histories were prepared. For sake of simplicity, the next sections will feature results related to a single one of these models (model IV in Ref. 80). This was produced according to the following protocol to quench the $\mathrm{GeSe}_{9}$ liquid: (a) the volume of the initial liquid configuration at $T=1000 \mathrm{~K}$ was modify to match the density of the glass, $(b)$ theGeSe 9 system was equilibrated at $T=1000 \mathrm{~K}$ for $10 \mathrm{ps}$, and (c) the thermostat was set to $T=900 \mathrm{~K}, T=600 \mathrm{~K}$ and $T=300 \mathrm{~K}$, with annealing over periods of time of 12.5 ps, 25 ps and 30 ps, respectively. Finally, statistical averages were extracted from each sub-trajectory along an interval of time of $30 \mathrm{ps}$ at $T=300 \mathrm{~K}$.

\section{Total neutron structure factor and total pair correlation function}

The total neutron structure factors $S_{\mathrm{T}}^{\mathrm{th}}(k)$ for glassy $\mathrm{Ge}_{2} \mathrm{Se}_{3}$ and glassy $\mathrm{GeSe}_{9}$ are presented in figure 14. In the left panel, the calculated neutron structure factor for $g-\mathrm{Ge}_{2} \mathrm{Se}_{3}$ is shown together with the corresponding experimental data, $S_{\mathrm{T}}^{\exp }(k)$ from Ref. 81 . The FPMD data are in very good agreement with the measurements, both position and intensity of the peaks being well reproduced over the full range of wavevectors.

In figure 14, right panel, the calculated FPMD total neutron structure factor $S_{\mathrm{T}}^{\text {th }}(k)$ for glassy GeSe 9 is shown along with two experimental counterparts: (a) the old measurement $S_{\mathrm{T}}^{\exp [\mathrm{a}]}(k)$ by Ramesh Rao etal from Ref. 82, and (b) the more recent measurements $S_{\mathrm{T}}^{\exp [\mathrm{b}]}(k)$ from Ref. 80. Both measurements exhibit a shoulder at $k \simeq 1.3 \AA^{-1}$, around the FSDP range of values, as expected for Ge-Se disordered materials. However, there is a difference between the two data sets in the low wave vector region. In Ref. 80 it was proposed that this large discrepancy stems from issues related to the background scattering in the neutron diffraction analysis. As illustrated in figure 14 , the calculated $S_{\mathrm{T}}^{\text {th }}(k)$ for $\mathrm{GeSe}_{9}$, is in very good agreement with $S_{\mathrm{T}}^{\exp [\mathrm{b}]}(k)$ measured in recent neutron diffraction experiments.

In diffraction experiments $S_{\mathrm{T}}(k)$ is usually Fourier transformed to obtain real space information in terms of the total pair correlation function $g_{\mathrm{T}}(r)$. Figure 15 shows the experimental total pair distribution function, $g_{\mathrm{T}}^{\exp }(r)$, obtained from reciprocal space data via Fourier transformation. The upper limits of integration are set to $k_{\max }=19.95 \AA^{-1}$ and $k_{\max }=30.0 \AA^{-1}$ for, respectively, $g-\mathrm{Ge}_{2} \mathrm{Se}_{3}$ and $g-\mathrm{GeSe}_{9}$. Due to the finite measurement window functions of the diffractometers used during these experiments, to which $k_{\max }$ corresponds, spurious oscillations are observed at 

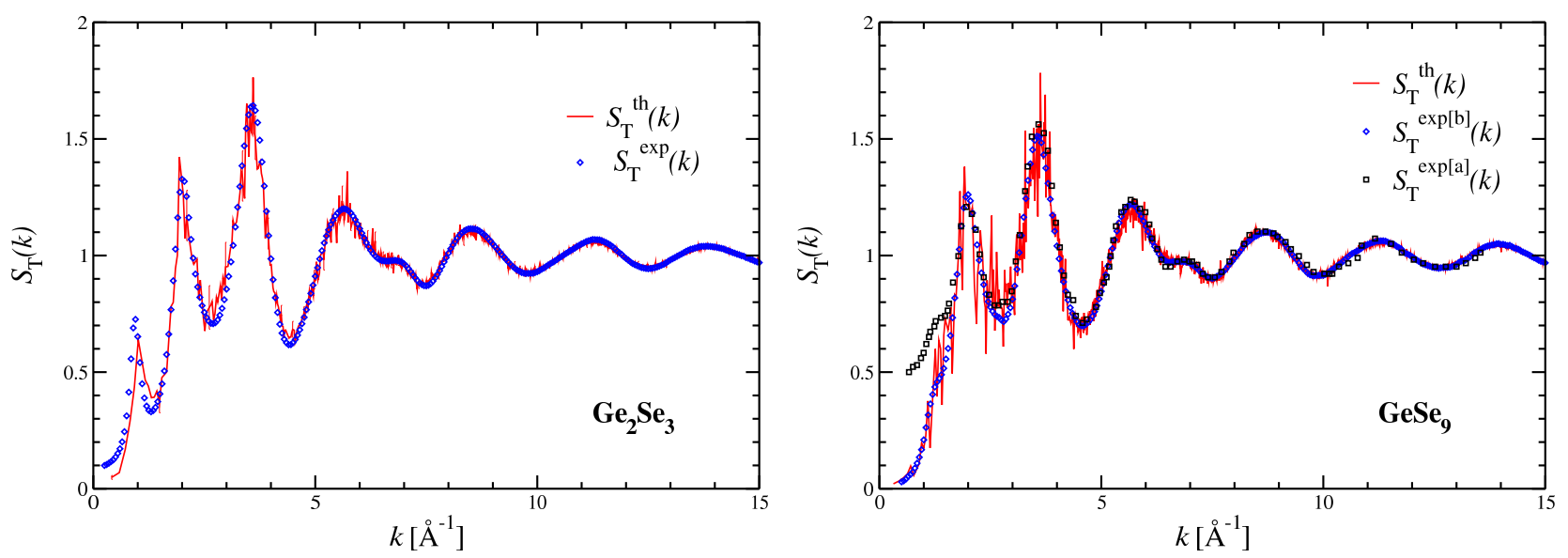

Figure 14: (Color online) Left: total neutron structure factor for glassy $\mathrm{Ge}_{2} \mathrm{Se}_{3}$ at $T=300 \mathrm{~K}$. Experimental data from Ref. 81, $S_{\mathrm{T}}^{\exp }(k)$ (red circles)are compared to the FPMD calculation result, $S_{\mathrm{T}}^{\text {th }}(k)$ (solid blue curve). Only a limited number of error bars is given in the reciprocal space.

Right: Total neutron structure factor for glassy $\mathrm{GeSe}_{9}$ at $T=300 \mathrm{~K}$. We present a comparison among the old experimental data from Ref. 82, $S_{\mathrm{T}}^{\exp [\mathrm{a}]}(k)$ (black squares), more recent measurements from Ref. 80, $S_{\mathrm{T}}^{\exp [\mathrm{b}]}(k)$ (blue diamonds) and the FPMD calculation result, $S_{\mathrm{T}}^{\mathrm{th}}(k)$ (solid red curve).

$r<2 \AA$. In addition, Figure 15 shows $g_{\mathrm{T}}^{\text {th }}(r)$ calculated by using the atomic coordinates of the model and $g_{\mathrm{T}}^{\mathrm{th}(\mathrm{b})}(r)$ calculated by following the experimental procedure (this meaning by Fourier transforming the calculated total structure factor $S_{\mathrm{T}}^{\mathrm{th}}(k)$ with $k_{\max }=19.95 \AA^{-1}$ for $\mathrm{Ge}_{2} \mathrm{Se}_{3}$ and $k_{\max }=30.0 \AA^{-1}$ for $\mathrm{GeSe}_{9}$ ). In figure 15 the right panel contains the results for $g-\mathrm{Ge}_{2} \mathrm{Se}_{3}$, while the left panel provides the results for $g-\mathrm{GeSe}_{9}$.

For both systems the first main peak of $g_{\mathrm{T}}^{\text {th }(\mathrm{b})}(r)$ is closer to $g_{\mathrm{T}}^{\exp }(r)$ than $g_{\mathrm{T}}^{\text {th }}(r)$. The total pair correlation function $g_{\mathrm{T}}^{\text {th }}(r)$ is not altered by the oscillations in the interval $2.5 \AA<r<3.5 \AA$.

For $g-\mathrm{Ge}_{2} \mathrm{Se}_{3}$, (figure 15 right panel), $g_{\mathrm{T}}^{\text {th }}(r)$ is very close to experiments when considering the intensity of the peak at $\sim 4 \AA$. The small bump at $5.5 \AA$ is very well reproduced by both $g_{\mathrm{T}}^{\text {th }}(r)$ and $g_{\mathrm{T}}^{\text {th }(\mathrm{b})}(r)$. For $g$-GeSe 9 , figure 15 left panel, $g_{\mathrm{T}}^{\text {th }}(r)$ features few differences with respect to the experimental data in between 4 and $6 \AA$, namely the intensity of the peak at $\sim 4 \AA$ is slightly smaller, and the position of the peak at $\sim 5.75 \AA$ is slightly shifted toward shorter $r$.

Overall, for both systems, the FPMD data agree well with experiments, confirming the very good capability of our modeling approach to describe quantitatively disordered $\mathrm{Ge}_{\mathrm{x}} \mathrm{Se}_{1-\mathrm{x}}$ materials. 

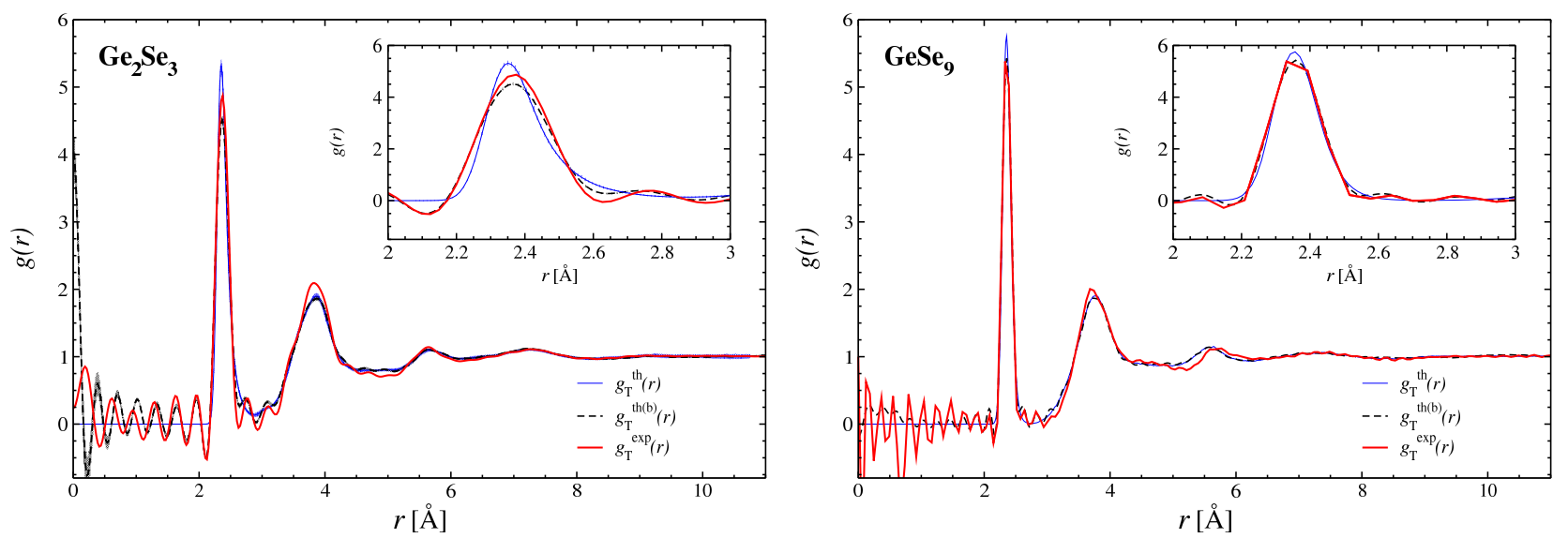

Figure 15: (Color online) Left: total pair correlation function for glassy $\mathrm{Ge}_{2} \mathrm{Se}_{3}$ at $T=300 \mathrm{~K}$. The experimental function $g_{\mathrm{T}}^{\exp }(r)$ is compared to the results from the FPMD calculation $g_{\mathrm{T}}^{\text {th }(\mathrm{b})}(r)$ and $g_{\mathrm{T}}^{\text {th }}(r) \cdot g_{\mathrm{T}}^{\exp }(r)$ is calculated by Fourier transforming the reciprocal space data $\left(S_{\mathrm{T}}^{\exp }(k)\right.$, see figure 14) from Ref. 81 with a cutoff value $k_{\max }=19.95 \AA^{-1} \cdot g_{\mathrm{T}}^{\text {th }(\mathrm{b})}(r)$ (broken black curve) was calculated using the same procedure to transform $S_{\mathrm{T}}^{\text {th }}(k)$ (see left panel in fig. 14). $g_{\mathrm{T}}^{\text {th }}(r)$ (solid blue curve) is the result of a direct calculation from the real-space coordinates. The inset corresponds to the region of the first peak, between 2 and $3 \AA$.

Right: total pair correlation function for glassy $\mathrm{GeSe}_{9}$ at $T=300 \mathrm{~K}$. The experimental function $g_{\mathrm{T}}^{\exp }(r)$ is compared to the results from the FPMD calculation $g_{\mathrm{T}}^{\text {th }(\mathrm{b})}(r)$ and $g_{\mathrm{T}}^{\text {th }}(r) \cdot g_{\mathrm{T}}^{\exp }(r)$ is calculated by Fourier transforming the reciprocal space data $\left(S_{\mathrm{T}}^{\exp [\mathrm{b}]}(k)\right.$, see right panel in fig. 14)from Ref. 80 with a cutoff value $k_{\max }=30.0 \AA^{-1} \cdot g_{\mathrm{T}}^{\text {th(b) }}(r)$ (broken black curve) was calculated using the same procedure to transform $S_{\mathrm{T}}^{\text {th }}(k)$ (see right panel in fig. 14). $g_{\mathrm{T}}^{\text {th }}(r)$ (solid blue curve) is calculated directly from the real-space coordinates. The inset correspond to the region of the first peak, between 2 and $3 \AA$. 


\section{Real space properties}

FPMD modeling offers the opportunity to gain information extracted directly from the atomic coordinates. For instance, partial pair correlation functions $g_{\alpha \beta}(r)$, the different coordination units and the bond angle distributions are frequently employed to describe with more precision the disordered network. 3D representations of two snapshots for glassy $\mathrm{Ge}_{2} \mathrm{Se}_{3}$ and glassy $\mathrm{GeSe}_{9}$ are shown in figure 16.

\section{Partial pair correlation functions}

Figure 17 shows the partial pair correlation functions $g_{\alpha \beta}(r)$ for $g-\mathrm{Ge}_{2} \mathrm{Se}_{3}$ (top) and $g$ - $\mathrm{GeSe}_{9}$ (bottom) calculated from the FPMD trajectories. Concerning the similarities between the two glasses, for both systems the main peak in $g_{\mathrm{GeSe}}(r)$ is quite sharp confirming the predominant presence of the Ge centered tetrahedron. In addition, $g_{\mathrm{GeGe}}(r)$ is characterized by two peaks, signatures of the presence of the basic building blocks of these networks: edge-sharing (ES) connections (peak at $\simeq 3.0 \AA$ ) and corner sharing $(\mathrm{CS})$ connections (peak at $\simeq 3.75 \AA$ ) within the tetrahedron. Furthermore, the peaks in $g_{\mathrm{SeSe}}(r)$ at $\simeq 4 \AA$ are typical signatures of the intra tetrahedral Se-Se distances.

When considering $g-\mathrm{Ge}_{2} \mathrm{Se}_{3}$ (figure 17 top panel), the partial pair correlation function $g_{\mathrm{SeSe}}(r)$ does not feature any mark at distances typical of $\mathrm{Se}-\mathrm{Se}$ homopolar bonds. On the other hand, $g_{\text {GeGe }}(r)$ is characterized by a sharp and intense peak at $\simeq 2.5 \AA$, indicative of Ge-Ge homopolar bonds, a strong signature of the $g-\mathrm{Ge}_{2} \mathrm{Se}_{3}$ network.

Turning to $g-\mathrm{GeSe}_{9}$ (figure 17 bottom panel), it appears that $g_{\mathrm{SeSe}}(r)$ is characterized by a sharp and intense peak at $\simeq 2.4 \AA$ due to the presence of Se-Se homopolar bonds, while $g_{\mathrm{GeGe}}(r)$ does not show any peak at short Ge-Ge distances.

Interestingly, both systems are characterized by a significant number of homopolar bonds, Ge-Ge for $\mathrm{Ge}_{2} \mathrm{Se}_{3}$ and, the opposite, Se-Se for $\mathrm{GeSe}_{9}$. This can be rationalized by considering that we are dealing with representative systems with an excess of either Se or Ge atoms with respect to the composition $x=0.33$. In one case, Ge atoms are in excess when all tetrahedra have been formed, in the other this same topological effect concerns Se atoms that arrange to bound to each other by forming extended Se chains. 

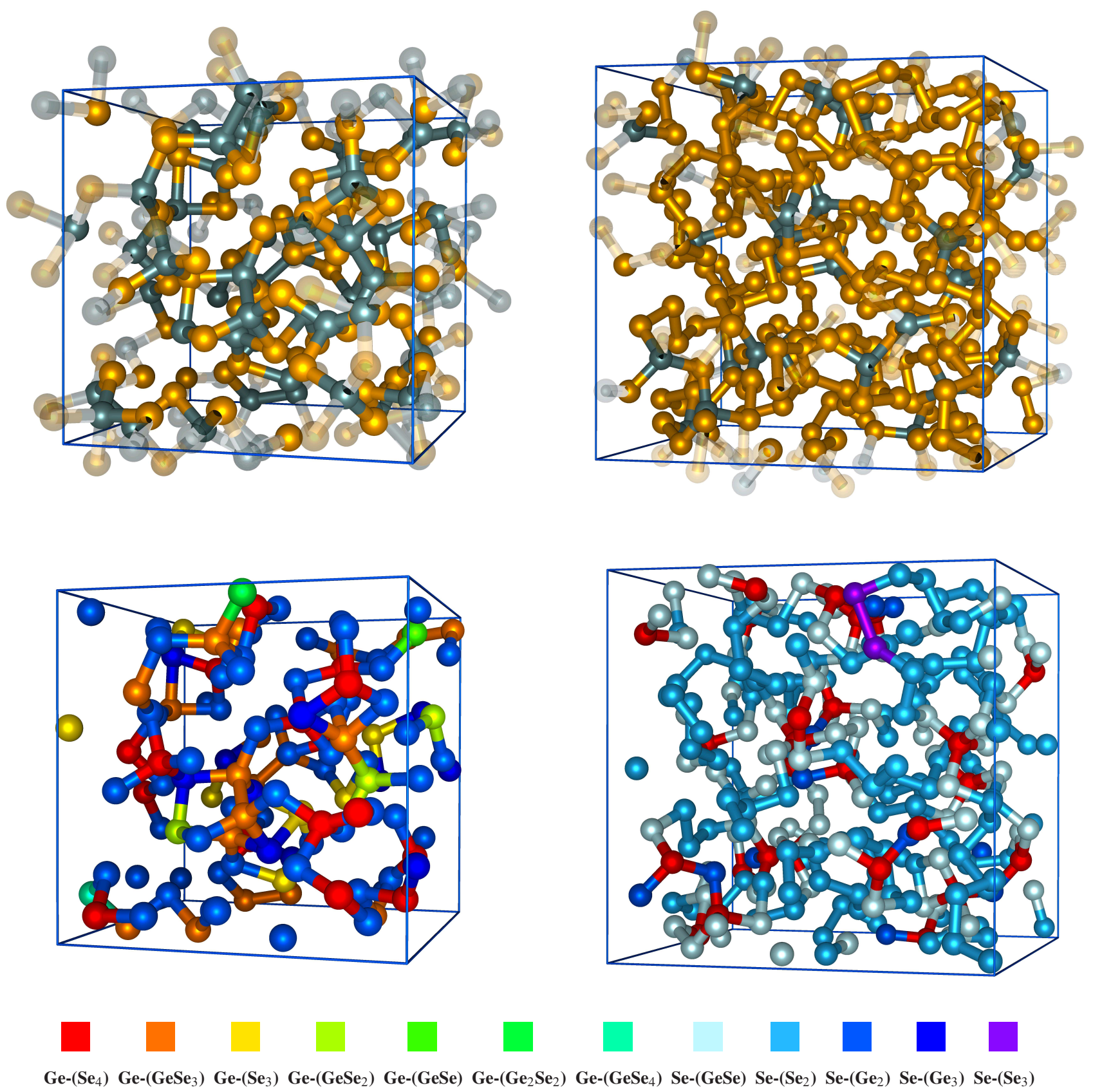

Figure 16: (Color online) Visual representations of the 3D models for $g-\mathrm{Ge}_{2} \mathrm{Se}_{3}$ (left) and $g$-GeSe 9 (right). Top: standard representation using the atomic species as colormap, Ge atoms are in dark (grey) color, Se atoms are in light (orange) color. Clones, i.e. atoms and bonds found using periodic boundary conditions are highlighted using transparency.

Bottom: representation using the partial coordinations as colormap, i.e. each different environment is highlighted with the colormap being the same for both glasses. For clarity clones are not shown. 


\section{Partial pair correlation functions $g_{\alpha \beta}(r)$}
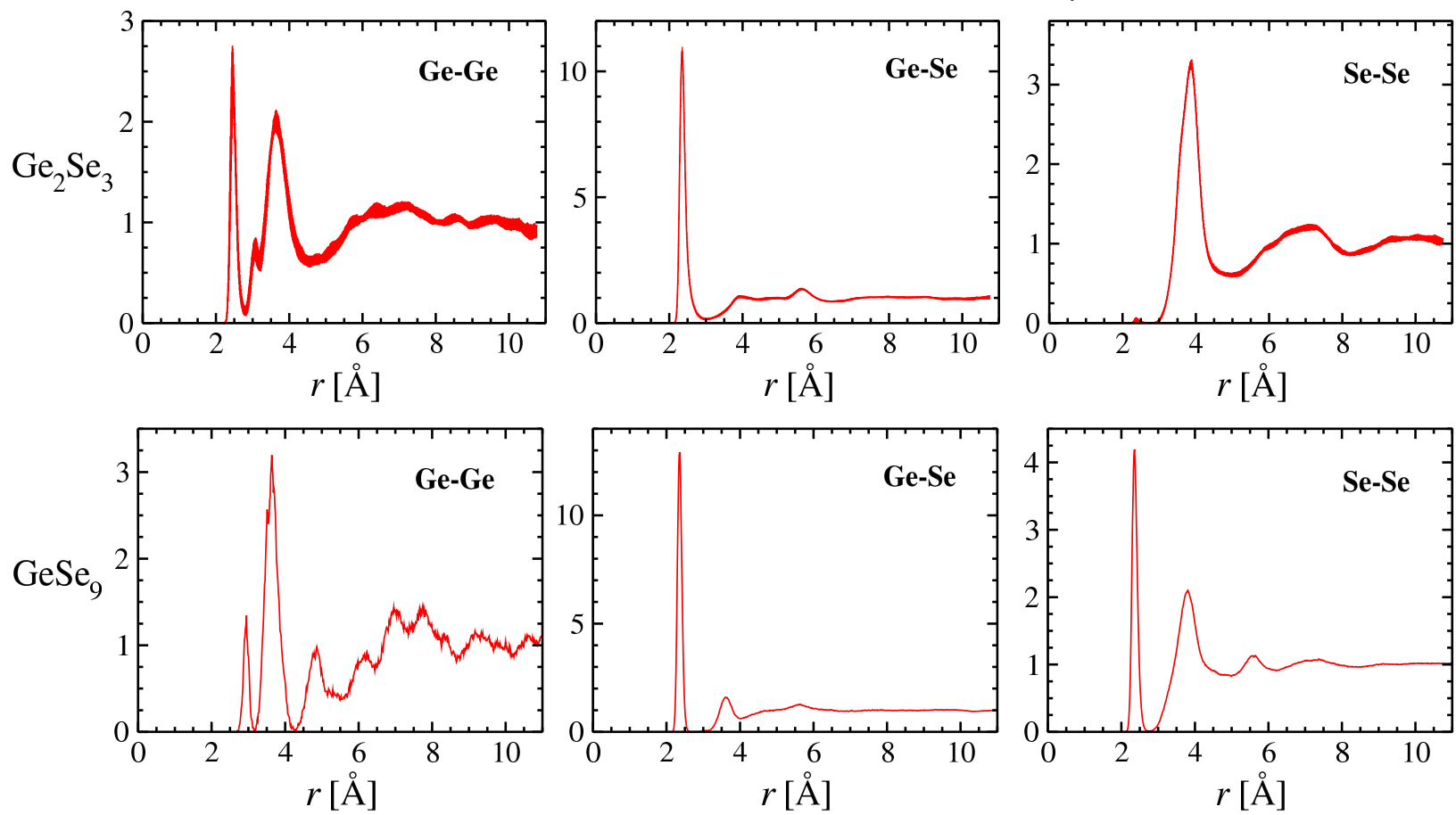

Figure 17: (Color online) Partial pair correlation functions $g_{\mathrm{GeGe}}(r)$ (left panel), $g_{\mathrm{GeSe}}(r)$ (middle panel) and $g_{\mathrm{SeSe}}(r)$ (right panel) for $g-\mathrm{Ge}_{2} \mathrm{Se}_{3}$ (top), and $g$-GeSe9 (bottom)

\section{Structural units and network topology}

The glassy structures of $\mathrm{GeSe}_{2}$ and $\mathrm{GeSe}_{9}$ can be analyzed in more detail by focusing on the building blocks, i.e. the $\alpha$ - $l$ structural units in which a given atom $\alpha$ (Ge or $\mathrm{Se}$ ) is $l$-fold connected to the nearest neighboring atoms. For a specific unit, $\bar{n}_{\alpha}(l)$, one calculates its mean number with respect to the total number of atoms of type $\alpha$. Following the results obtained for the total pair correlation function, $g_{\mathrm{T}}(r)$, we considered that bonds are formed when the distance between a given pair of atoms does not exceed $2.9 \AA$ that is the first minimum in $g_{\mathrm{T}}(r)$. This choice is consistent with the analysis carried out for other binary chalcogenide systems [12, 83, 84]. The number percentage of $l$-fold coordinated atoms and the number occurrence of each specific unit $\bar{n}_{\alpha}(l)$ in $g-\mathrm{Ge}_{2} \mathrm{Se}_{3}$ and $g-\mathrm{GeSe}_{9}$ are presented in figure 18. A visual illustration of this analysis using the results as colormap superposed to a standard 3D representation of the $g-\mathrm{Ge}_{2} \mathrm{Se}_{3}$ and $g$-GeSe 9 models is made available in figure 16 .

The first information to be extracted from figure 18 concerns the proportion of the two main coordination units for each atomic species in the two glasses. In $g-\mathrm{Ge}_{2} \mathrm{Se}_{3}$ the fourfold $\mathrm{Ge}$ atoms 
(74.7\%), and the twofold Se atoms $(84.7 \%)$ dominate, while in $g$-GeSe 9 both atomic species Ge and Se are almost exclusively found in their standard coordination (>99.9\%), fourfold and twofold respectively. In $g-\mathrm{Ge}_{2} \mathrm{Se}_{3}$ the majority of the twofold $\mathrm{Se}$ atoms are linked to two Ge atoms $(84 \%)$, the expected conformation for this species. The fourfold Ge atoms are divided between standard $\mathrm{Ge}-\left(\mathrm{Se}_{4}\right)(35.7 \%)$ and miscoordinated $\mathrm{Ge}-\left(\mathrm{GeSe}_{3}\right)(33.4 \%)$ atoms. Indeed, the large number of $\mathrm{Ge}$ atoms in $g-\mathrm{Ge}_{2} \mathrm{Se}_{3}$ leads to a departure from a perfect tetrahedral network, almost half of the fourfold Ge atoms being at the center of $\mathrm{Se}_{3} \mathrm{Ge}$ pseudo-tetrahedra. In $g$-GeSe 9 essentially all of the Ge atoms are found in their tetrahedral Ge-( $\left.\mathrm{Se}_{4}\right)$ conformation. While the Se atoms are mostly twofold, the proportions of Se-( $\left.\mathrm{Se}_{2}\right)$ and $\mathrm{Se}-(\mathrm{GeSe})$ coordination units become very significant. Also, the proportion of Se- $\left(\mathrm{Ge}_{2}\right)$ units decreases dramatically.
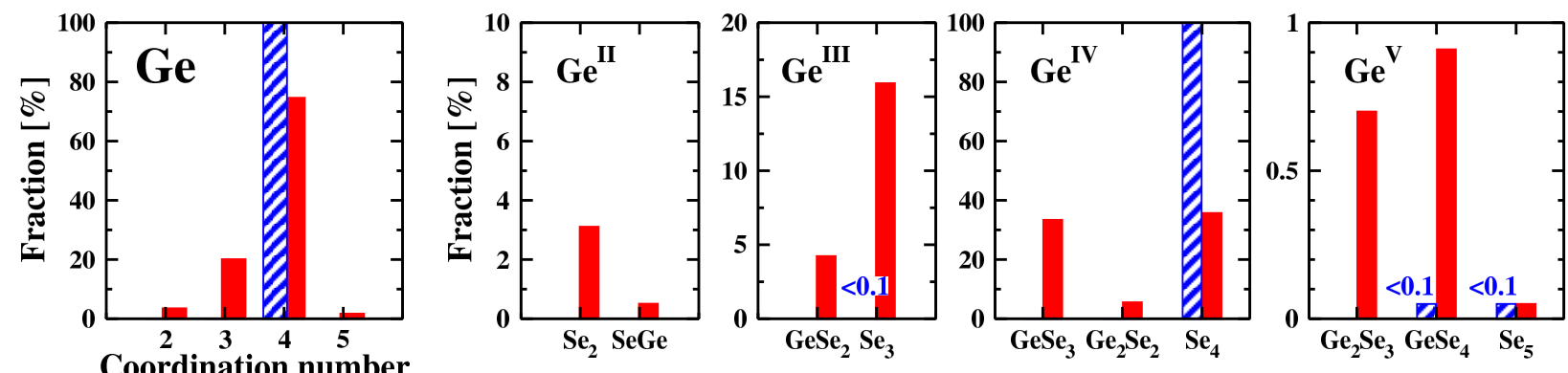

$g-\mathrm{Ge}_{2} \mathrm{Se}_{3}$
$\mathrm{~g}-\mathrm{GeSe}_{9}$
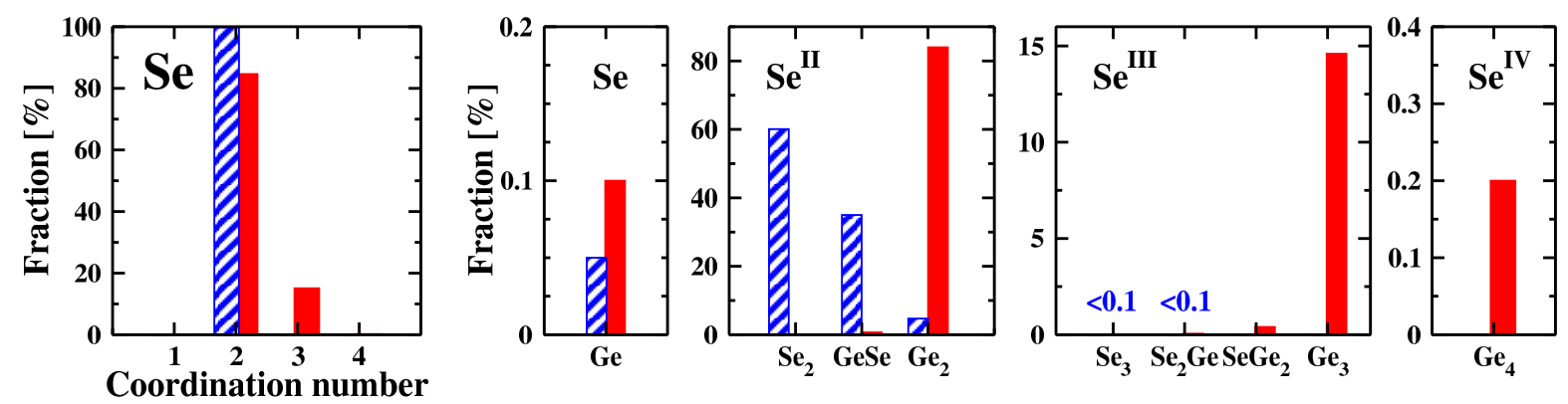

Figure 18: (Color online) Percentage of $l$-fold coordinated atoms, also decomposed in term of each specific unit $\bar{n}_{\alpha}(l)$, for both $\mathrm{Ge}$ (top panel) and Se (bottom panel) in $g-\mathrm{Ge}_{2} \mathrm{Se}_{3}$ (red) and $g-\mathrm{GeSe}_{9}$ (blue with dashes). 


\section{Bond angles distribution}

The tetrahedral nature of these networks is also highlighted by the bond angle distributions $\theta_{\mathrm{GeSeGe}}$ and $\theta_{\mathrm{SeGeSe}}$ shown in figure 19 (left panel). The quantity $\theta_{\mathrm{GeSeGe}}$ features the expected peaks around $80^{\circ} \pm 3$ and $100^{\circ} \pm 2$, these being signatures of the basic building blocks of the amorphous network in the $\mathrm{Ge}_{\mathrm{x}} \mathrm{Se}_{1-\mathrm{x}}$ family, namely the ES and CS tetrahedra respectively (see Fig. 19 right panel) [12]. In $g-\mathrm{GeSe}_{9}$ these two peaks are separated by a well with a vanishing minimum, i.e. no Ge-Se-Ge angles are found in $g-\mathrm{GeSe}_{9}$ around $\simeq 85^{\circ}$. This is different from the case of $g-\mathrm{Ge}_{2} \mathrm{Se}_{3}$ for which $\mathrm{Ge}-\mathrm{Se}-\mathrm{Ge}$ angles have values spanning the entire $70-140^{\circ}$ range, as visible in the left panel of figure 19. An explanation lies in the lack of Ge-Ge homopolar bonds in $g$-GeSe 9 . Indeed Ge-Ge homopolar bonds, that are particularly important in $g$ - $\mathrm{Ge}_{2} \mathrm{Se}_{3}$, are likely to disrupt the geometry of standard tetrahedral $\mathrm{Ge}\left(\mathrm{Se}_{4}\right)$ geometry, in particular in the case of the $\mathrm{Ge}\left(\mathrm{Se}_{3} \mathrm{Ge}\right)$ coordination units.

Accordingly, one finds that $\theta_{\mathrm{SeGeSe}}$ has a peak at $109^{\circ}$, the expected value of the tetrahedral angle, for both glasses.

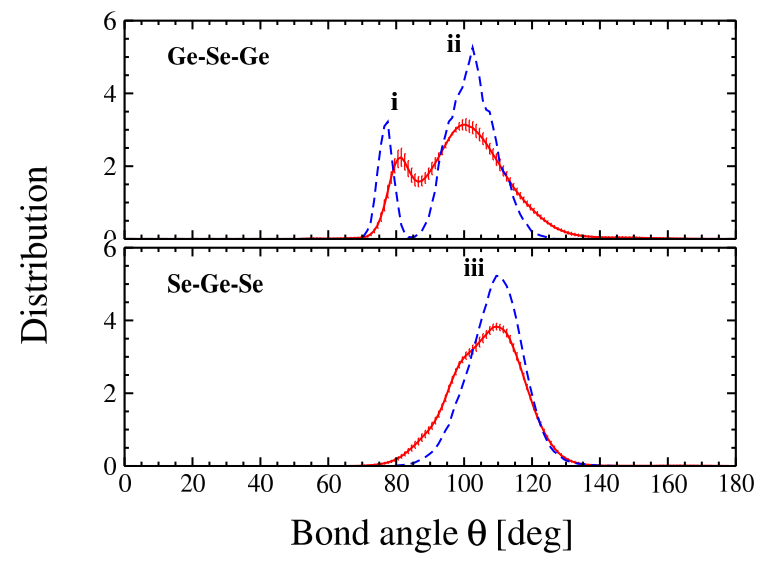

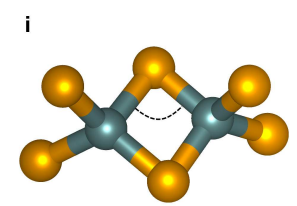

iii

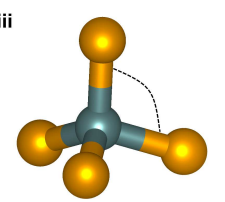

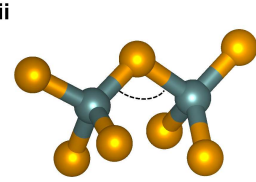

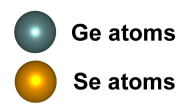

Figure 19: (Color online) Left: Bond-angle distributions for $g-\mathrm{Ge}_{2} \mathrm{Se}_{3}$ (red curve) and $g$-GeSe 9 (blue dashes) $\theta_{\mathrm{GeSeGe}}$ (top panel) and $\theta_{\mathrm{SeGeSe}}$ (bottom panel).

Right: the corresponding coordination units in the $\mathrm{Ge}_{\mathrm{x}} \mathrm{Se}_{1-\mathrm{x}}$ family of disordered materials.

\section{Summary and conclusions}

This review has provided an account of accomplishments obtained in the area of first principles modeling of disordered chalcogenide systems. These materials proved to be particularly challeng- 
ing for atomic-scale approaches due to the non-trivial iono-covalent character of their bonding and electronic properties. We began by showing how FPMD can be used to contribute to the understanding of a generic property, by conferring a quantitative character to calculations impacting an entire class of systems, those featuring intermediate-range order. This led us to focus on the atomic-scale origins (in direct space) of the first sharp diffraction peak exhibited by certain structure factors in reciprocal space. Then, we turned to the description of specific cases, each one bearing an interest both in terms of methodology and of information collected on the atomic structure of chalcogenide systems. Glassy $\mathrm{GeSe}_{2}$ is a revealing example of stoichiometric network with a moderate and yet sizeable departure from chemical order, taking the form of a (small) number of $\mathrm{Ge}(\mathrm{Se})$ atoms not being fourfold (twofold) coordinated. The case of glassy $\mathrm{SiSe}_{2}$ was instrumental to underline the impact of first principles periodic cell calculations (as opposed to cluster calculations) to achieve quantitative comparison with experimental data concerning the relative weight of specific structural motifs. As a third case of network system at the stoichiometric concentration, glassy $\mathrm{GeS}_{2}$ gave us the opportunity to improve upon previous results obtained by using non-self consistent density functional methods. We also applied to glassy $\mathrm{GeS}_{2}$ a methodology (the Wannier function localization scheme) capable of differentiating systems of equal concentration on the basis of their degree of ionicity. Our interest in glassy $\mathrm{GeSe}_{4}$ was motivated by a longstanding debate on its atomic structure, since the presence of Se chains could be compatible, in principle, with a $\mathrm{Se}_{n} / \mathrm{GeSe}_{2}$ phase separation. By using FPMD we were able to demonstrate that such a phase separation does not occur. In a further section, we have reviewed the behavior of glassy $\mathrm{GeSe}_{2}$ and glassy $\mathrm{GeSe}_{4}$ under the action of an external pressure causing enhanced densification. Due to the different topology of the Se sub-network in the absence of pressure, totally different structures are found when pressure is applied. In the first (glassy $\mathrm{GeSe}_{2}$ ), one observes a transition from the tetrahedral to the octahedral arrangement. In the second (glassy $\mathrm{GeSe}_{4}$ ), the Se chains are able to adjust to the overall reduction of available volume, by preserving the tetrahedral structure. We have pursued our review with an example of amorphous ternary system $\left(\mathrm{Ga}_{4} \mathrm{Sb}_{6} \mathrm{Te}_{3}\right)$ to mark our growing interest for phase change materials relevant in the area of memory devices and optical disks.

In terms of methodology, we found worthwhile to present an example of study, devoted to glassy $\mathrm{GeTe}_{4}$, based on a set of calculations differing by the inclusion (or the neglect) of dispersion forces, combined with different expressions for the exchange-correlation functional. This specific section ends with a warning on the different performances of two alternative recipes for 
the dispersion forces, adapting in various degrees to changes of the electronic structure. Also on the methodological side, we report on a comparative classical MD/FPMD study carried out by taking advantage of an interatomic potential devised for liquid $\mathrm{GeSe}_{2}$. It appears that the classical description performs reasonably well in terms of structural properties. However, it leads to totally different diffusive properties, in line with the absence of homopolar bonds that cannot occur since short nearest-neighbor interatomic distances between atoms of the same kind are not compatible with their bearing charges of equal sign.

On the side of recent advances in terms of applications, we have reported very recent results on the calculation of thermal conductivity, by exploiting a scheme well adapted to first-principles molecular dynamics. We have shown, for the case of glassy $\mathrm{GeTe}_{4}$, under which conditions one can obtain very realistic values for the thermal conductivity. Finally, the review ends with the detailed account of the structural properties of two binary chalcogenide glassy materials, $\mathrm{GeSe}_{9}$ and $\mathrm{Ge}_{2} \mathrm{Se}_{3}$, located at opposite sides in the concentration range of the $\mathrm{Ge}_{\mathrm{x}} \mathrm{Se}_{1-\mathrm{x}}$ family.

Throughout this review, we showed that first-principles molecular dynamics is a very useful tool to describe disordered systems (chalcogenide glasses in particular) and gather information on their structural arrangement. Spectacular increase in computational power and the reliability of theoretical recipes have made possible the achievement of calculations in excellent agreement with experiments. However, FPMD calculations do not have to be considered as an accessible and handy route to be taken inevitably in the search of a plausible atomic-scale description. Experience collected in the case of chalcogenides has instructed us on the multiples pitfalls one might encounter when using FPMD without considering properly crucial ingredients. As such we can mention the adequate length of the equilibrium trajectories, the size of the periodic box in the presence of intermediate range correlations and of extended phonon paths when describing thermal transport, the kind of exchange-correlation functional, the role of dispersion forces and the treatment of data when switching from direct to reciprocal space (Fourier transform vs. direct calculation). Users have become more and more aware of these possible drawbacks, thereby granting to FPMD the rank of technique popular among theoreticians willing to capture with a quantitative approach the atomic-scale details and mechanisms underlying the properties of materials.

[1] T. E. Faber and Z. J. M., Phil. Mag. 11, 153 (1965).

[2] A. B. Bhatia and D. E. Thornton, Phys. Rev. B 2, 3004 (1970). 
[3] C. Massobrio, A. Pasquarello, and R. Car, Phys. Rev. Lett. 80, 2342 (1998).

[4] J. P. Perdew and Y. Wang, Phys. Rev. B 45, 13244 (1992).

[5] C. Massobrio, A. Pasquarello, and R. Car, J. Am. Chem. Soc. 121, 2943 (1999).

[6] A. D. Becke, Phys. Rev. A 38, 3098 (1988).

[7] C. Lee, W. Yang, and R. G. Parr, Phys. Rev. B 37, 785 (1988).

[8] http://www.cpmd.org, Copyright jointly by IBM Corp. and by Max Planck Institute, Stuttgart. (20002019).

[9] J. A. White and D. M. Bird, Phys. Rev. B 50, 4954 (1994).

[10] D. M. Bylander and L. Kleinman, J. Comp. Phys. 136, 599 (1997).

[11] C. Massobrio and A. Pasquarello, J. Chem. Phys. 114, 7976 (2001).

[12] C. Massobrio, A. Pasquarello, and R. Car, Phys. Rev. B 64, 144205 (2001).

[13] M. Wilson and P. A. Madden, Phys. Rev. Lett. 80, 532 (1998).

[14] S. R. Elliott, Phys. Rev. Lett. 67, 711 (1991).

[15] C. Massobrio and A. Pasquarello, Phys. Rev. B 75, 014206 (2007).

[16] I. T. Penfold and P. S. Salmon, Phys. Rev. Lett. 67, 97 (1991).

[17] M. Boero, A. Bouzid, S. Le Roux, B. Özdamar, and C. Massobrio, Molecular Dynamics Simulations of Disordered Materials: From Network Glasses to Phase-Change Memory Alloys (Springer, 2015).

[18] M. Boero and A. Oshiyama, Car-Parrinello Molecular Dynamics in Encyclopedia of Nanotechnology (Springer, 2015).

[19] L. Giacomazzi, C. Massobrio, and A. Pasquarello, Phys. Rev. B 75, 174207 (2007).

[20] C. Massobrio and A. Pasquarello, Phys. Rev. B 77, 144207 (2008).

[21] I. Petri, P. S. Salmon, and H. E. Fischer, Phys. Rev. Lett. 84, 2413 (2000).

[22] A. Bouzid and C. Massobrio, J. Chem. Phys. 137, 046101 (2012).

[23] M. Celino and C. Massobrio, Phys. Rev. Lett. 90, 125502 (2003).

[24] K. Jackson and S. Grossman, Phys. Rev. B 65, 012206 (2001).

[25] K. Sykina, E. Furet, B. Bureau, S. Le Roux, and C. Massobrio, Chem. Phys. Lett. 547, 30 (2012).

[26] J. P. Perdew, K. Burke, and M. Ernzerhof, Phys. Rev. Lett. 77, 3865 (1996).

[27] M. Celino, S. Le Roux, G. Ori, B. Coasne, A. Bouzid, M. Boero, and C. Massobrio, Phys. Rev. B 88, 174201 (2013).

[28] S. Blaineau, P. Jund, and D. A. Drabold, Phys. Rev. B 67, 094204 (2003).

[29] S. Blaineau and P. Jund, Phys. Rev. B 69, 064201 (2004). 
[30] S. Le Roux and P. Jund, J. Phys.: Cond. Mat. 19, 169102 (2007).

[31] O. F. Sankey and D. J. Niklewski, Phys. Rev. B 40, 3979 (1989).

[32] J. Harris, Phys. Rev. B 31, 1770 (1985).

[33] E. Bychkov, M. Fourmentin, M. M., and C. Benmore (2001), p. 54.

[34] A. Zeidler, W. E. Drewitt, P. S. Salmon, A. C. Barnes, W. A. Crichton, S. Klotz, H. E. Fischer, C. J. Benmore, S. Ramos, and A. C. Hannon, J. Phys.: Cond. Mat. 21, 474217 (2009).

[35] N. Marzari and D. Vanderbilt, Phys. Rev. B 56 (1997).

[36] G. H. Wannier, Phys. Rev. B 52 (1937).

[37] K. Wezka, A. Bouzid, K. J. Pizzey, P. S. Salmon, A. Zeidler, S. Klotz, H. E. Fischer, C. L. Bull, M. G. Tucker, M. Boero, et al., Phys. Rev. B 90, 054206 (2014).

[38] K. Wezka, P. S. Salmon, A. Zeidler, D. A. J. Whittaker, J. W. E. Drewitt, S. Klotz, H. E. Fischer, and D. Marrocchelli, J. Phys.: Cond. Mat. 24, 502101 (2012).

[39] L. P. Dávila, M.-J. Caturla, A. Kubota, B. Sadigh, T. Díaz de la Rubia, J. F. Shackelford, S. H. Risbud, and S. H. Garofalini, Phys. Rev. Lett. 91, 205501 (2003).

[40] S. K. Lee, P. J. ENG, H. K. Mao, Y. Meng, M. Newville, M. Y. Hu, and J. Shu, Nature (London) . Mat. 4, 851 (2005).

[41] Y. Liang, C. R. Miranda, and S. Scandolo, Phys. Rev. B 75, 024205 (2007).

[42] D. Marrocchelli, M. Salanne, and P. A. Madden, J. Phys.: Cond. Mat. 22, 152102 (2010).

[43] A. Zeidler, K. Wezka, D. A. J. Whittaker, P. S. Salmon, A. Baroni, , S. Klotz, H. E. Fischer, M. C. Wilding, C. L. Bull, et al., Phys. Rev. B 90, 024206 (2014).

[44] A. Zeidler, K. Wezka, R. F. Rowlands, D. A. J. Whittaker, P. S. Salmon, A. Polori, J. W. E. Drewitt, S. Klotz, H. E. Fischer, , et al., Phys. Rev. Lett. 113, 135501 (2014).

[45] M. Wilson and P. S. Salmon, Phys. Rev. Lett. 103, 157801 (2009).

[46] A. Bouzid, K. J. Pizzey, A. Zeidler, G. Ori, M. Boero, C. Massobrio, S. Klotz, H. E. Fischer, C. L. Bull, and P. S. Salmon, Phys. Rev. B 93, 014202 (2016).

[47] L. B. Skinner, C. J. Benmore, S. Antao, E. Soignard, S. A. Amin, E. Bychkov, E. Rissi, J. B. Parise, and J. L. Yarger, J. Phys. Chem. C 116, 2212 (2012).

[48] B. Kalkan, R. P. Dias, C.-S. Yoo, S. M. Clark, and S. Sen, J. Phys. Chem. C 118, 5110 (2014).

[49] A. Bouzid, S. Gabardi, C. Massobrio, M. Boero, and M. Bernasconi, Phys. Rev. B 91, 184201 (2015).

[50] M. Wuttig and N. Yamada, Nature Materials 6 (2007).

[51] J. H. Los, T. D. Küne, G. S., and M. Bernasconi, Phys. Rev. B 88, 174203 (2013). 
[52] S. Maurugeon, B. Bureau, C. Boussard-Plédel, F. A., P. Lucas, X. H. Zhang, and J. Lucas, Optical Materials 33, 660 (2011).

[53] P. Lucas, C. Boussard-Plédel, A. Wilhelm, S. Danto, X. H. Zhang, P. Houizot, S. Maurugeon, B. Conseil, and B. Bureau, Fibers 1, 110 (2013).

[54] L. Run, T. Shi-Yu, B. Gang, Y. Qiao-Nan, L. Xue-Xin, X. Yi-Dong, Y. Jiang, and L. Zhi-Guo, Chin. Phys. Lett. 30, 058101 (2013).

[55] H. Jiang, K. Guo, H. Xu, Y. Xia, K. Jiang, F. Tang, J. Yin, and L. Z., J. Appl. Phys. 109, 066104 (2011).

[56] M. Micoulaut, J. Chem. Phys. 138, 061103 (2013).

[57] A. Bouzid, C. Massobrio, M. Boero, G. Ori, K. Sykina, and E. Furet, Phys. Rev. B 92, 134208 (2015).

[58] S. Grimme, J. Comp. Chem. 27, 1787 (2006).

[59] C. Massobrio, M. Micoulaut, and P. S. Salmon, Solid State Sci. 12, 199 (2010).

[60] M. Micoulaut, R. Vuilleumier, and C. Massobrio, Phys. Rev. B 79, 214205 (2009).

[61] L. Giacomazzi, C. Massobrio, and A. Pasquarello, J. Phys.: Cond. Mat. 23, 295401 (2011).

[62] I. Kaban, T. Halm, W. Hoyer, P. Jovari, and J. Neuefeind, J. Non-Cryst. Solids. 326, 120 (2003).

[63] P. Silvestrelli, Phys. Rev. Lett. 100, 053020 (2008).

[64] E. Lampin, A. Bouzid, G. Ori, M. Boero, and C. Massobrio, J. Chem. Phys. 147, 044504 (2017).

[65] C. Massobrio, E. Martin, Z. Chaker, M. Boero, A. Bouzid, S. Le Roux, and G. Ori, Frontiers in Materials 5, 78 (2018).

[66] M. Wilson, B. K. Sharma, and C. Massobrio, J. Chem. Phys. 128, 244505 (2008).

[67] P. A. Madden and M. Wilson, Chem. Soc. Rev. 25, 339 (1996).

[68] B. K. Sharma and M. Wilson, Phys. Rev. B 73, 060201 (2006).

[69] E. Lampin, P. L. Palla, P.-A. Francioso, and F. Cleri, J. Appl. Phys. 114, 033525 (2013).

[70] A. Sommerfeld, Lectures on Theoretical Physics, Partial Differential Equations in Physics, vol.6 (Academic Press, 1949).

[71] S.-N. Zhang, J. He, T.-J. Zhu, X.-B. Zhao, and T. Tritt, J. Non-Cryst. Solids. 355, 79 (2009).

[72] H. Zaoui, P. L. Palla, F. Cleri, and E. Lampin, Phys. Rev. B 94, 054304 (2016).

[73] P. S. Salmon, J. Non-Cryst. Solids. 353, 2959 (2007).

[74] R. Car and M. Parrinello, Phys. Rev. Lett. 55, 2471 (1985).

[75] N. Troullier and J. L. Martins, Phys. Rev. B 43, 1993 (1991).

[76] S. Nosé, Mol. Phys. 52, 255 (1984). 
[77] W. G. Hoover, Phys. Rev. A 31, 1695 (1985).

[78] P. E. Blöchl and M. Parrinello, Phys. Rev. B 45, 9413 (1992).

[79] S. Le Roux, A. Zeidler, P. S. Salmon, M. Boero, M. Micoulaut, and C. Massobrio, Phys. Rev. B 84, $134203(2011)$.

[80] S. Le Roux, A. Bouzid, K. Y. Kim, S. Han, A. Zeidler, P. S. Salmon, and C. Massobrio, J. Chem. Phys. 145, 084502 (2016).

[81] P. S. Salmon and J. Liu, J. Phys.: Cond. Mat. 6, 1449 (1994).

[82] N. R. Rao, P. S. R. Krishna, S. Basu, B. A. Dasannacharya, K. S. Sangunni, and E. S. R. Gopal, J. Non-Cryst. Solids. 240, 221 (1998).

[83] M. J. Haye, C. Massobrio, A. Pasquarello, A. De Vita, S. W. De Leeuw, and R. Car, Phys. Rev. B 58, R14661 (1998).

[84] F. H. M. van Roon, C. Massobrio, E. de Wolff, and S. W. de Leeuw, J. Chem. Phys. 113, 5425 (2000). 